\title{
PETSc Developers Guide
}

Revision 3.9

Mathematics and Computer Science Division 


\section{About Argonne National Laboratory}

Argonne is a U.S. Department of Energy laboratory managed by UChicago Argonne, LLC under contract DE-AC02-06CH11357. The Laboratory's main facility is outside Chicago, at 9700 South Cass Avenue, Argonne, Illinois 60439. For information about Argonne and its pioneering science and technology programs, see www.anl.gov.

\section{DOCUMENT AVAILABILITY}

Online Access: U.S. Department of Energy (DOE) reports produced after 1991 and a growing number of pre-1991 documents are available free at OSTI.GOV (http://www.osti.gov/), a service of the US Dept. of Energy's Office of Scientific and Technical Information.

Reports not in digital format may be purchased by the public from the National Technical Information Service (NTIS):

U.S. Department of Commerce

National Technical Information Service

5301 Shawnee Rd

Alexandria, VA 22312

www.ntis.gov

Phone: (800) 553-NTIS (6847) or (703) 605-6000

Fax: (703) 605-6900

Email: orders@ntis.gov

Reports not in digital format are available to DOE and DOE contractors from the Office of Scientific and Technical Information (OSTI):

U.S. Department of Energy

Office of Scientific and Technical Information

P.O. Box 62

Oak Ridge, TN 37831-0062

www.osti.gov

Phone: (865) 576-8401

Fax: (865) 576-5728

Email: reports@osti.gov

\section{Disclaimer}

This report was prepared as an account of work sponsored by an agency of the United States Government. Neither the United States Government nor any agency thereof, nor UChicago Argonne, LLC, nor any of their employees or officers, makes any warranty, express or implied, or assumes any legal liability or responsibility for the accuracy, completeness, or usefulness of any information, apparatus, product, or process disclosed, or represents that its use would not infringe privately owned rights. Reference herein to any specific commercial product, process, or service by trade name, trademark, manufacturer, or otherwise, does not necessarily constitute or imply its endorsement, recommendation, or favoring by the United States Government or any agency thereof. The views and opinions of document authors expressed herein do not necessarily state or reflect those of the United States Government or any agency thereof, Argonne National Laboratory, or UChicago Argonne, LLC. 
ANL-18/18 Rev 3.9

\section{PETSc Developers Manual}

Revision 3.9

Prepared by

S. Kruger ${ }^{1}$, P. Sanan ${ }^{2}$, and B. Smith ${ }^{3}$

${ }^{1}$ Tech-X Corporation

${ }^{2}$ Institute of Geophysics, ETH Zurich

${ }^{3}$ Mathematics and Computer Science Division, Argonne National Laboratory

June 2018

This material was based upon work supported by the Office of Science, Office of Advanced Scientific Computing Research,

U.S. Department of Energy, under Contract DE-AC02-06CH11357. 



\begin{abstract}
PETSc is an extensible software library for scientific computation. This document provides information for PETSc developers and those wishing to contribute to PETSc. The text assumes that you are familiar with PETSc and have access to PETSc source code and documentation (available via http://www.mcs.anl.gov/petsc) including the PETSc users manual [2]. Higher-level views of PETSc can be found in [10], [1], [8], [5], and [4].

Before contributing code to PETSc, please read Chapter 2, which contains the source code style guide. Information on how to submit patches and pull requests to PETSc can be found at http://www.mcs.anl.gov/petsc/developers/index.html.
\end{abstract}

Please direct all comments and questions regarding PETSc design and development to petscdev@mcs.anl.gov. Note that all bug reports and questions regarding the use of PETSc should be directed to petsc-maint@mcs.anl.gov. 



\section{Contents}

1 Responding to User Requests and Questions 99

2 Style Guide $\quad 10$

2.1 Names . . . . . . . . . . . . . . . . . . . . . . . . . . . 10

2.2 Coding Conventions and Style . . . . . . . . . . . . . . . 11

2.2 .1 C Formatting . . . . . . . . . . . . . . . . . . . 11

2.2 .2 C Usage . . . . . . . . . . . . . . . . . . . . . . . . 13

2.2.3 Usage of PETSc Functions and Macros . . . . . . . . . . . . . . . . . 14

2.3 Formatted Comments . . . . . . . . . . . . . . . . . 15

3 The PETSc Kernel $\quad 18$

3.1 PETSc Types . . . . . . . . . . . . . . . . . . . 18

3.2 Implementation of Error Handling . . . . . . . . . . . . . . . . . . . . . 19

3.2 .1 Simplified Interface . . . . . . . . . . . . . . . . . . . 19

3.2 .2 Error Handlers . . . . . . . . . . . . . . . . . . . . . . . . . 19

3.2 .3 Error Codes . . . . . . . . . . . . . . . . . . 20

3.2 .4 Detailed Error Messages . . . . . . . . . . . . . . . . . . . 20

3.3 Memory Management . . . . . . . . . . . . . . . . . . . . . . . . . . . . . . . . . . .

3.4 Implementation of Profiling . . . . . . . . . . . . . . . . . . 22

3.4.1 Profiling Object Creation and Destruction . . . . . . . . . . . . . . 22

3.4 .2 Profiling Events . . . . . . . . . . . . . . . . . . 22

3.4.3 Controlling Profiling . . . . . . . . . . . . . . . . . . 22

4 Basic Object Design and Implementation 23

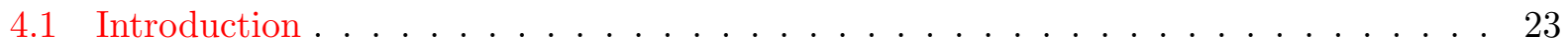

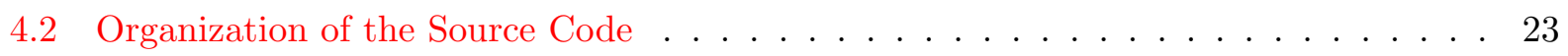

4.3 Common Object Header . . . . . . . . . . . . . . . . . . . . . 24

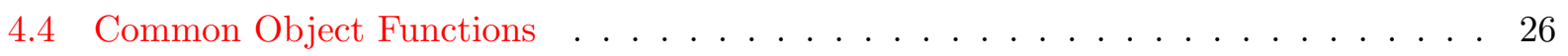

4.5 Object Function Implementation . . . . . . . . . . . . . . . . . . . . . . . . . .

4.5.1 Compose and Query Objects . . . . . . . . . . . . . . 27

4.5 .2 Compose and Query Functions . . . . . . . . . . . . . . . . . . . . . . . . . . . . . 28

4.5.3 Simple PETSc Objects. . . . . . . . . . . . . . . . 29

4.6 PETSc Packages . . . . . . . . . . . . . . . . . . . . 29

5 How the Solvers Handle User Provided Callbacks $\quad 30$ 
6 The Various Matrix Classes $\quad 35$

6.1 Matrix Blocking Strategies . . . . . . . . . . . . . . . . . . 35

6.2 Assorted Matrix Types . . . . . . . . . . . . . . . . . 36

6.2 .1 Sequential AIJ Sparse Matrices . . . . . . . . . . . . . . . . 36

6.2.2 Parallel AIJ Sparse Matrices . . . . . . . . . . . . . . . . . . . 36

6.2 .3 Sequential Block AIJ Sparse Matrices . . . . . . . . . . . . . . . . . . 37

6.2 .4 Parallel Block AIJ Sparse Matrices . . . . . . . . . . . . . . . . . . . 37

6.2.5 Sequential Dense Matrices . . . . . . . . . . . . . . . . . . 37

6.2 .6 Parallel Dense Matrices . . . . . . . . . . . . . . . . . . 37

7 PETSc Testing System $\quad 38$

7.1 PETSc Test Description Language . . . . . . . . . . . . . . . . . . . . 38

7.1 .1 Runtime Language Options . . . . . . . . . . . . . . . . . . . . 39

7.1 .2 Additional Specifications . . . . . . . . . . . . . . . . 41

7.1 .3 Test Block Examples . . . . . . . . . . . . . . . . . . . . . . 41

7.1 .4 Build Language Options . . . . . . . . . . . . . . . . . . . . . . . . . . . . . . . . . . . . . .

7.2 PETSC Test Harness . . . . . . . . . . . . . . . . . . . . . . . . 44

7.3 Test Output Standards: TAP . . . . . . . . . . . . . . . . . . . 44

7.4 Test Harness Implementation ． . . . . . . . . . . . . . . . . . 45

7.5 Using the Test Harness for Regression Testing . . . . . . . . . . . . . . . . . . 46

7.5 .1 Additional Tips . . . . . . . . . . . . . . . . 47 


\section{Chapter 1}

\section{Responding to User Requests and Questions}

PETSc users communicate to the PETSc team via two mailing lists: petsc-maint@mcs.anl.gov and petsc-users@mcs.anl.gov. When responding to such inquiries, please follow the guidelines given below.

- Be polite.

- Address the person by name (when you can determine the name).

- Apologize for the problem when it is appropriate (but not otherwise).

- Thank the person for his or her patience if it is more than six hours since the report came in.

- If the person drops the petsc-maint or petsc-users from the reply list, add it back in.

- Don't ask too many questions or give too many suggestions in the same email. The user often responds only to the first of them or becomes confused. 


\section{Chapter 2}

\section{Style Guide}

The PETSc team uses certain conventions to make the source code consistent and hence easier to maintain. We will interchangeably use the terminology subclass, implementation, or type ${ }^{1}$ to refer to a concrete realization of an abstract base class. For example, KSPGMRES is a type for the base class KSP.

\subsection{Names}

Consistency of names for variables, functions, and so on is extremely important. We use several conventions

1. All function names and enum types consist of acronyms or words, each of which is capitalized, for example, KSPSolve() and MatGetOrdering().

2. All enum elements and macro variables are named with all capital letters. When they consist of several complete words, there is an underscore between each word. For example, MAT_FINAL_ASSEMBLY.

3. Functions that are private to PETSc (not callable by the application code) either

- have an appended_Private (for example, StashValues_Private) or

- have an appended_Subtype (for example, MatMult_SeqAIJ).

In addition, functions that are not intended for use outside of a particular file are declared static. Also see item 15 in Section 2.2.3.

4. Function names in structures (for example, _matops) are the same as the base application function name without the object prefix and are in small letters. For example, MatMultTranspose() has a structure name of multtranspose.

5. Names of implementations of class functions should begin with the function name, an underscore, and the name of the implementation, for example, KSPSolve_GMRES().

6. Each application-usable function begins with the name of the class object, followed by any subclass name, for example, ISInvertPermutation(), MatMult(), or KSPGMRESSetRestart().

7. Functions that PETSc provides as defaults for user-providable functions end with Default (for example, KSPMonitorDefault() or PetscSignalHandlerDefault()).

\footnotetext{
${ }^{1}$ Type also refers to the string name of the subclass.
} 
8. Each application usable function begins with the name of the class object, followed by any subclass name, for example, MatMult() or KSPGMRESSetRestart().

9. Options database keys are lower case, have an underscore between words, and match the function name associated with the option without the word "set" or "get", for example, -ksp_gmres_restart.

10. Specific XXXType values (for example, MATSEQAIJ) do not have an underscore in them unless they refer to another package that uses an underscore, for example, MATSOLVERSUPERLU_DIST.

\subsection{Coding Conventions and Style}

Within the PETSc source code, we adhere to the following guidelines so that the code is uniform and easily maintained.

\subsubsection{Formatting}

1. No tabs are allowed in any of the source code.

2. All PETSc function bodies are indented two characters.

3. Each additional level of loops, if statements, and so on is indented two more characters.

4. Wrapping lines should be avoided whenever possible.

5. Source code lines do not have a hard length limit; generally, we like them less than 150 characters wide.

6. The local variable declarations should be aligned. For example, use the style

PetscScalar a;

PetscInt i, j;

instead of

PetscScalar a;

PetscInt $i, j ; / *$ Incorrect $* /$

7. Assignment and comparison operations, for example, $\mathrm{x}=22.0$ or $\mathrm{x}<22.0$, should have single spaces around the operator. This convention is true even when assignments are given directly in a line that declares the variable, such as PetscReal $r=22.3$. The exception is when these symbols are used in a for loop; then, there should be no spaces, for example, for $(i=0 ; i<m ; i++)$. Comparisons in while() constructs should have the spaces.

8. When declaring variables there should be no space between multiple variables, for example, PetscReal a,b,c, not PetscReal a, b, c.

9. The prototypes for functions should not include the names of the variables; for example, write PETSC_EXTERN PetscErrorCode MyFunction(PetscInt);

not

PETSC_EXTERN PetscErrorCode MyFunction(PetscInt myvalue); /* Incorrect */ 
10. All local variables of a particular type (for example, PetscInt) should be listed on the same line if possible; otherwise, they should be listed on adjacent lines.

11. Equal signs should be aligned in regions where possible.

12. There must be a single blank line between the local variable declarations and the body of the function.

13. Indentation for if statements must be done as follows.

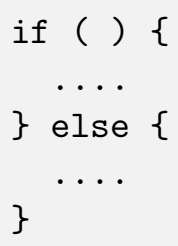

14. Never have

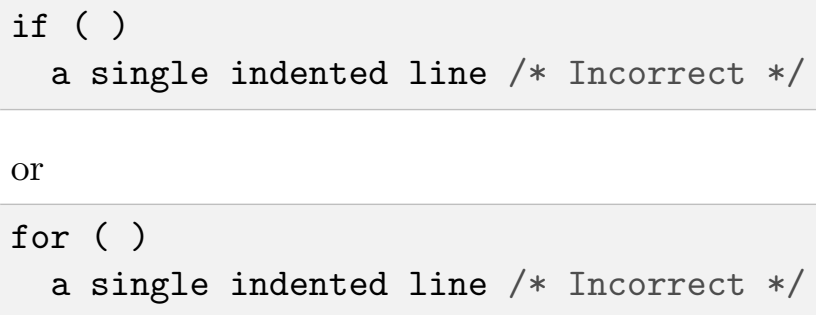

or

Instead, use either

if ( ) a single statement

or

if ( ) \{

a single indented line

\}

Note that error checking is a separate statement, so the following is incorrect

if ( ) ierr $=\operatorname{XXX}() ; \operatorname{CHKERRQ(ierr);~} / *$ Incorrect $* /$

and instead you should use

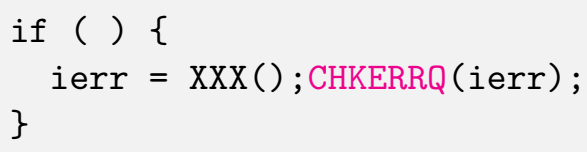

15. Always have a space between if or for and the following ().

16. The open brace should be on the same line as the if ( ) test, for ( ), and so forth, not on its own line, for example,

$$
\text { \} else }\{
$$

instead of 


\section{\}}

else $\{/ *$ Incorrect $* /$

See item 17 for an exception. The closing brace should always be on its own line.

17. In function declarations, the opening brace should be on the next line, not on the same line as the function name and arguments. This is an exception to item 16.

18. Do not leave sections of commented-out code in the source files.

19. Do not use $\mathrm{C}++$-style comments (// Comment). Use only C-style comments (/* Comment $* /)$.

20. All variables must be declared at the beginning of the code block (C89 style), never mixed in with code.

21. Do not include a space after a ( or before a ). Do not write

ierr $=$ PetscMalloc1 $(10, \& a) ; \operatorname{CHKERRQ(ierr);~} / *$ Incorrect $* /$

but instead write

ierr = PetscMalloc1 $(10$, \&a) ; CHKERRQ (ierr);

22. Do not use a space after the ) in a cast or between the type and the $*$ in a cast.

23. Do not include a space before or after a comma in lists. That is, do not write

ierr $=$ func $(\mathrm{a}, 22.0) ; \operatorname{CHKERRQ}($ ierr $) ; / *$ Incorrect $* /$

but instead write

ierr $=$ func $(a, 22.0) ;$ CHKERRQ (ierr);

24. The format strings in PETSc ASCI output routines, such as PetscPrintf, take a \%D for all PETSc variables of type PetscInt, not a \%d.

25. All arguments of type PetscReal to PETSc ASCI output routines, such as PetscPrintf, must be cast to double, for example,

PetscPrintf(PETSC_COMM_WORLD, " Norm \%g\n' , (double)norm);

\subsubsection{Usage}

1. Array and pointer arguments where the array values are not changed should be labeled as const arguments.

2. Scalar values passed to functions should never be labeled as const.

3. Subroutines that would normally have a void** argument to return a pointer to some data should actually be prototyped as void*. This prevents the caller from having to put a (void**) cast in each function call. See, for example, DMDAVecGetArray(). 
4. Do not use the register directive.

5. Do not use if ( $r a n k==0)$ or if ( $\mathrm{v}==$ NULL) or if ( $\mathrm{flg}==$ PETSC_TRUE) or if ( $\mathrm{flg}$ $==$ PETSC_FALSE). Instead, use if ( $\mathrm{rank})$ or if $(\mathrm{l} \mathrm{v})$ or if $(\mathrm{flg})$ or if $(! \mathrm{flg})$.

6. Do not use \#ifdef or \#ifndef. Rather, use \#if defined(... or \#if !defined(...

7. Never use system random number generators such as rand() in PETSc code or examples because these can produce different results on different systems thus making portability testing difficult. Instead use the PetscRandom which produces the exact same results regardless of system it is used on.

\subsubsection{Usage of PETSc Functions and Macros}

1. Public PETSc include files, petsc*.h, should not reference private PETSc petsc/private/ *impl.h include files.

2. Public and private PETSc include files cannot reference include files located in the PETSc source tree.

3. The first line of the executable statements in functions must be PetscFunctionBegin;

4. Use PetscFunctionReturn(returnvalue), not return(returnvalue);

5. Never put a function call in a return statement; do not write

PetscFunctionReturn( $\operatorname{somefunction}(\ldots)$ ) ; /* Incorrect */

6. Do not put a blank line immediately after PetscFunctionBegin; or a blank line immediately before PetscFunctionReturn(0); .

7. Do not use sqrt(), pow(), sin(), and so on directly in PETSc C/C++ source code or examples (usage is fine in Fortran source code). Rather, use PetscSqrtScalar(), PetscSqrtReal(), and so on, depending on the context. See petscmath.h for expressions to use.

8. Do not include assert.h in PETSc source code. Do not use assert(), it doesn't play well in the parallel MPI world.

9. The macros SETERRQ() and CHKERRQ() should be on the same line as the routine to be checked unless doing so violates the 150 character-width-rule. Try to make error messages short but informative.

10. Do not include a space before $\operatorname{CHKXXX}()$. That is, do not write

ierr $=$ PetscMalloc1 $(10, \& a) ; \operatorname{CHKERRQ(ierr);/*~Incorrect~} * /$

but instead write

ierr $=$ PetscMalloc1 $(10$, \&a $) ;$ CHKERRQ (ierr);

11. Except in code that may be called before PETSc is fully initialized, always use PetscMallocN() (for example, PetscMalloc1()), PetscCallocN(), PetscNew(), and PetscFree(), not malloc() and free(). 
12. MPI routines and macros that are not part of the 1.0 or 1.1 standard should not be used in PETSc without appropriate ./configure checks and \#if defined() checks. Code should also be provided that works if the MPI feature is not available, for example,

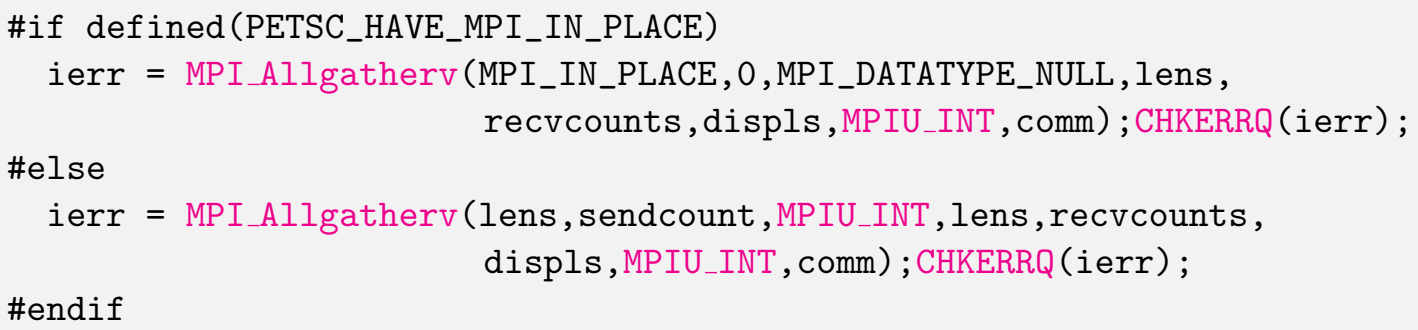

13. Do not introduce PETSc routines that provide essentially the same functionality as an available MPI routine. For example, do not write a routine PetscGlobalSum() that takes a scalar value and performs an MPI_Allreduce() on it. Instead, use the MPI routine MPI_Allreduce() directly in the code.

14. Never use a local variable counter such as PetscInt flops $=0$; to accumulate flops and then call PetscLogFlops(); always just call PetscLogFlops() directly when needed.

15. Library functions should be declared PETSC_INTERN if they are intended to be visible only within a single PETSc shared library. They should be declared PETSC_EXTERN if intended to be visible across shared libraries. Note that PETSc can be configured to build a separate shared library for each top-level class (Mat, Vec, KSP, and so on) and that plugin implementations of these classes can be included as separate shared libraries; thus, private functions may need to be marked PETSC_EXTERN. For example,

- MatStashCreate_Private is marked PETSC_INTERN as it is used across compilation units, but only within the Mat package;

- all functions, such as KSPCreate(), included in the public headers (include/petsc*.h) should be marked PETSC_EXTERN;

- MatHeaderReplace() is not intended for users (it is in include/petsc/private/matimpl. h) but is marked PETSC_EXTERN since it is used both by implementations of the Mat class (which could be defined in plugin implementations) and by functions in the DM and KSP packages.

16. Before removing or renaming an API function or type, PETSC_DEPRECATED () should be used in the relevant header file to indicate the new, correct usage and the version number where the deprecation will first appear. For example,

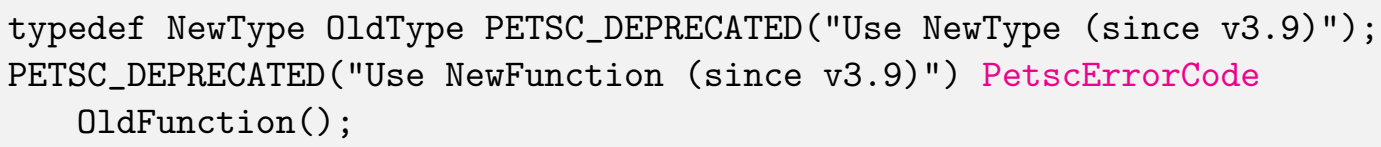

The old function or type, with the deprecation warning, should remain for at least one major release. The function or type's manual page should be updated (see 2.3, item 8).

\subsection{Formatted Comments}

PETSc uses formatted comments and the Sowing packages $[6,7]$ to generate documentation (manual pages) and the Fortran interfaces. Documentation for Sowing and the formatting may be found at 
http://wgropp.cs.illinois.edu/projects/software/sowing/; in particular, see the documentation for doctext.

- $/ * Q$

a formatted comment of a function that will be used for both documentation and a Fortran interface.

- $/ * @ \mathrm{C}$

a formatted comment of a function that will be used only for documentation, not to generate a Fortran interface. In general, such labeled $\mathrm{C}$ functions should have a custom Fortran interface provided. Functions that take char* or function pointer arguments must have the $\mathrm{C}$ symbol and a custom Fortran interface provided.

- $/ * \mathrm{E}$

a formatted comment of an enum used for documentation only. Note that each of these needs to be listed in lib/petsc/conf/bfort-petsc.txt as a native and defined in the corresponding include/petsc/finclude/petscxxx.h Fortran include file and the values set as parameters in the file src/SECTION/f90-mod/petscSUBSECTION.h, for example, src/vec/f90mod/petscis.h.

- $/ * S$

a formatted comment for a data type such as KSP. Note that each of these needs to be listed in $\mathrm{lib} /$ petsc/conf/bfort-petsc.txt as a nativeptr.

- $/ * \mathrm{MC}$

a formatted comment of a CPP macro or enum value for documentation.

The Fortran interface files supplied by the user go into the two directories ftn-custom and f90-custom, while those generated by Sowing go into ftn-auto.

\section{Manual Page Format}

Each function, typedef, class, macro, enum, and so on in the public API should include the following data, correctly formatted (see codes section) to generate complete manual pages and Fortran interfaces with Sowing. All entries below should be separated by blank lines. Except where noted, add a newline after the section headings.

1. The item's name, followed by a dash and brief (one-sentence) description.

2. If documenting a function, a description of the function's "collectivity" (whether all ranks in an MPI communicator need to participate).

- Not Collective if the function need not be called on all MPI ranks

- Collective [on $\mathrm{XXX}$ ] if the function is a collective operation (with respect to the data of class XXX)

- Logically Collective [on $\mathrm{XxX}$ ] if the function is collective but does not require any actual synchronization (say, setting class parameters uniformly).

3. If documenting a function with input parameters, a list of input parameter descriptions in an Input Parameters: section.

4. If documenting a function with output parameters, a list of output parameter descriptions in an Output Parameters: section. 
5. If documenting a function that interacts with the options database, a list of options database keys in an Options Database Keys: section.

6. (Optional) a Notes section containing in-depth discussion, technical caveats, special cases, and so on. If it is ambiguous whether returned pointers/objects need to be freed/destroyed by the user or not, this information should be mentioned here.

7. (If applicable) a Fortran Notes : section detailing any relevant differences in calling or using the item from Fortran.

8. Level: (no newline) followed by beginner, intermediate, advanced, developer, or deprecated.

9. (Optional) Concepts: (no newline), followed by a list of concepts.

10. (Optional) Keywords: (no newline), followed by a list of keywords.

11. . seealso: (no newline), followed by a list of related manual pages. These manual pages should usually also point back to this manual page in their seealso: sections. 


\section{Chapter 3}

\section{The PETSc Kernel}

PETSc provides a variety of basic services for writing scalable, component-based libraries; these are referred to as the PETSc kernel [5]. The source code for the kernel is in src/sys. It contains systematic support for

- managing PETSc types,

- error handling,

- memory management,

- profiling,

- object management,

- Fortran interfaces (see [3])

- mechanism for generating appropriate citations for algorithms and software used in PETSc (see [9])

- file $\mathrm{I} / \mathrm{O}$,

- an options database, and

- objects and code for viewing, drawing, and displaying data and solver objects.

Each of these is discussed in a section below.

\subsection{PETSc Types}

For maximum flexibility, the basic data types int, double, and so on are not used in PETSc source code. Rather, it has

- PetscScalar,

- PetscInt,

- PetscMPIInt,

- PetscBlasint,

- PetscBool, and

- PetscBT - bit storage of logical true and false.

PetscInt can be set using ./configure to be either int (32 bit, the default) or long long (64 bit, with configure --with-64-bit-indices) to allow indexing into very large arrays. PetscMPIInt is used for integers passed to MPI as counts and sizes. These are always int since that is what the MPI standard uses. Similarly, PetscBLASInt is for counts, and so on passed to BLAS and 
LAPACK routines. These are almost always int unless one is using a special "64-bit integer" BLAS/LAPACK (this is available, for example, with Intel's MKL and OpenBLAS).

In addition, there are special types:

- PetscClassId

- PetscErrorCode

- PetscLogEvent

These are currently always int, but their use clarifies the code.

\subsection{Implementation of Error Handling}

PETSc uses a "call error handler; then (depending on result) return error code" model when problems are detected in the running code. The public include file for error handling is include/ petscerror.h, and the source code for the PETSc error handling is in src/sys/error/.

\subsubsection{Simplified Interface}

The simplified macro-based interface consists of the following two calls:

- SETERRQ (comm, error code, "Error message");

- CHKERRQ (ierr);

The macro SETERRQ() is given by

return PetscError (comm,__LINE_-, PETSC_FUNCTION_NAME,__FILE__, error code,PETSC_ERROR_INITIAL, "Error message");

It calls the error handler with the current function name and location: line number, and file, plus an error code and an error message. Normally comm is PETSC_COMM_SELF; it can be another communicator only if one is absolutely sure the same error will be generated on all processes in the communicator. This feature is to prevent the same error message from being printed by many processes.

The macro CHKERRQ() is defined by

if (ierr) PetscError(PETSC_COMM_SELF,__LINE_-,PETSC_FUNCTION_NAME_,__FILE__, ierr, PETSC_ERROR_REPEAT, " ");

In addition to SETERRQ(), the macros SETERRQ1(), SETERRQ2(), SETERRQ3(), and SETERRQ4() allow one to provide additional arguments to a formatted message string, for example, SETERRQ2 (comm, PETSC_ERR, "Iteration overflow: its \%D norm \%g", its, (double)norm);

The reason for the numbered format is that C89 CPP macros cannot handle a variable number of arguments.

\subsubsection{Error Handlers}

The error-handling function PetscError() calls the "current" error handler with the code

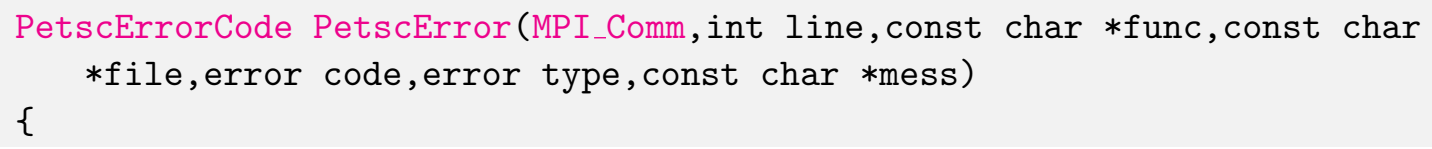




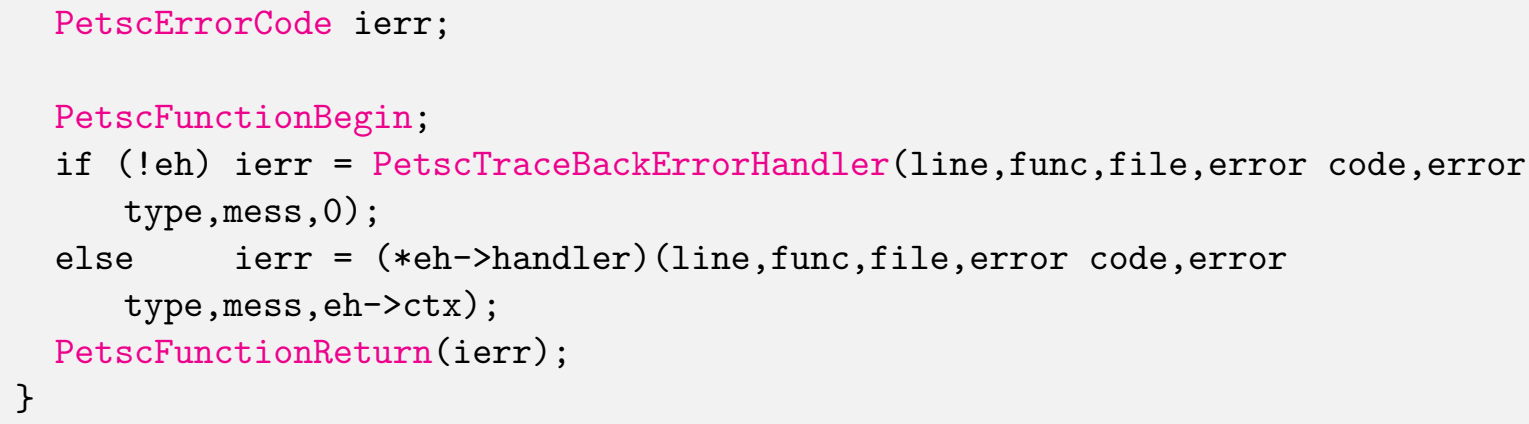

The variable eh is the current error handler context and is defined in src/sys/error/err.c as

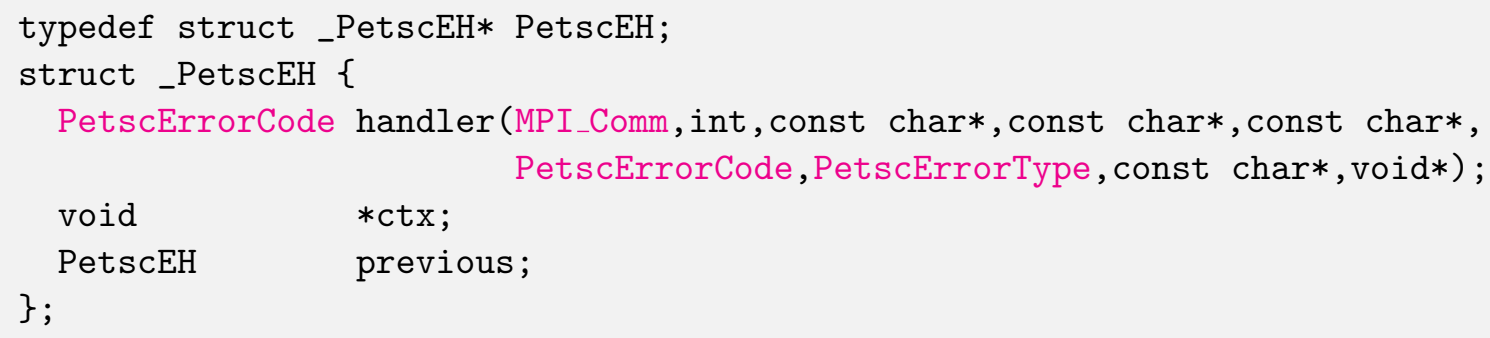

You can set a new error handler with the command PetscPushErrorHandler(), which maintains a linked list of error handlers. The most recent error handler is removed via PetscPopErrorHandler ().

PETSc provides several default error handlers:

- PetscTraceBackErrorHandler(), the default;

- PetscAbortErrorHandler(), called with -on_error_abort, useful when running in the debugger;

- PetscReturnErrorHandler(), which returns up the stack without printing error messages;

- PetscEmacsClientErrorHandler();

- PetscMPIAbortErrorHandler(), which calls MPI_Abort() after printing the error message; and

- PetscAttachDebuggerErrorHandler(), called with -on_error_attach_debugger.

\subsubsection{Error Codes}

The PETSc error handler takes an error code. The generic error codes are defined in include/ petscerror.h. The same error code is used many times in the libraries. For example, the error code PETSC_ERR_MEM is used whenever a requested memory allocation is not available.

\subsubsection{Detailed Error Messages}

In a modern parallel component-oriented application code, it does not always make sense to simply print error messages to the terminal (and more than likely there is no "terminal", for example, with Microsoft Windows or Apple iPad applications). PETSc provides the replaceable function pointer (*PetscErrorPrintf) ("Format" , ...);

which, by default, prints to standard out. Thus, error messages should not be printed with printf () or fprintf(). Rather, they should be printed with (*PetscErrorPrintf)(). You can direct all error messages to stderr, instead of the default stdout, with the command line option error_output_stderr. 


\subsection{Memory Management}

PETSc provides simple wrappers for the system malloc(), calloc(), and free() routines. The public interface for these is provided in petscsys. $\mathrm{h}$, while the implementation code is in src/sys/memory. The most basic interfaces are

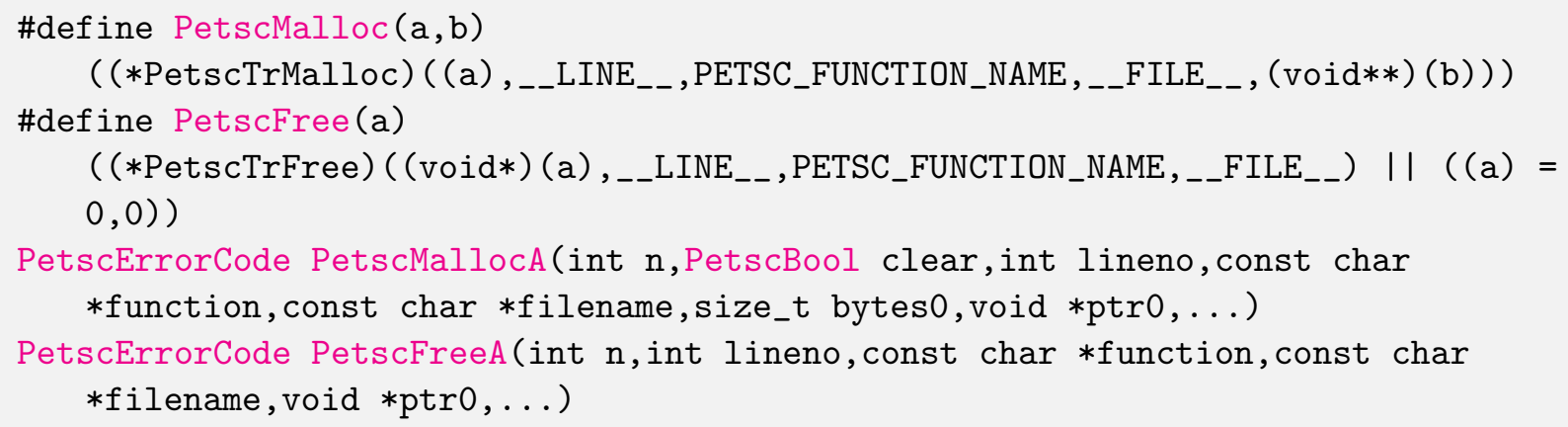

which allow the use of any number of profiling and error-checking wrappers for malloc(), $\operatorname{calloc}()$, and free(). Both PetscMallocA() and PetscFreeA() call the function pointer values $(*$ PetscTrMalloc) and (*PetscTrFree). PetscMallocSet () is used to set these function pointers. The functions are guaranteed to support requests for zero bytes of memory correctly. Freeing memory locations also sets the pointer value to zero, preventing later code from accidently using memory that has been freed. All PETSc memory allocation calls are memory aligned on at least double-precision boundaries; the macro generated by configure PETSC_MEMALIGN indicates in bytes what alignment all allocations have. This can be controlled at configure time with the option -with-memalign $=<4,8,16,32,64>$.

PetscMallocA() supports a request for up to 7 distinct memory locations of possibly different types. This serves two purposes: it reduces the number of system malloc () calls, thus potentially increasing performance, and it clarifies in the code related memory allocations that should be freed together.

The following macros are the preferred way to obtain and release memory in the PETSc source code. They automatically manage calling PetscMallocA() and PetscFreeA() with the appropriate location information.

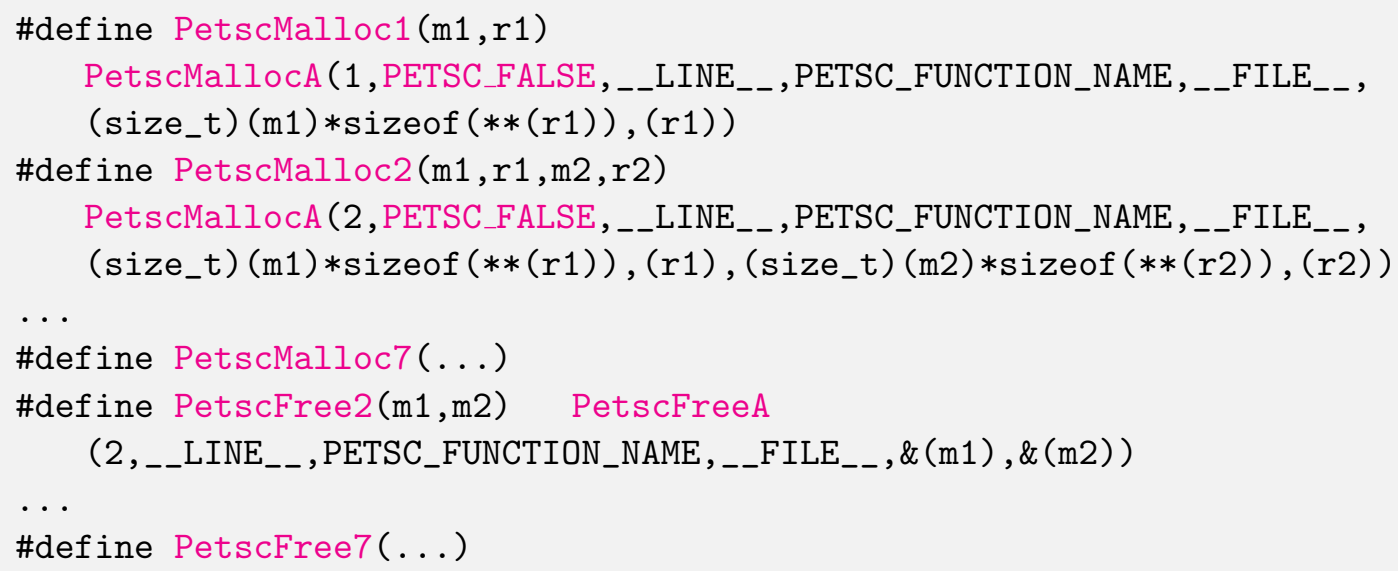

Similar routines, PetscCalloc1() to PetscCalloc7(), provide memory initialized to zero. The size requests for these macros are in number of data items requested, not in bytes. This decreases the number of errors in the code since the compiler determines their sizes from the object type instead of requiring the user to provide the correct value with sizeof (). 
The routines PetscTrMallocDefault() and PetscTrFreeDefault(), which are set with the routine PetscSetUseTrMalloc_Private() (and are used by default for the debug version of PETSc), provide simple logging and error checking versions of memory allocation.

\subsection{Implementation of Profiling}

This section provides details about the implementation of event logging and profiling within the PETSc kernel. The interface for profiling in PETSc is contained in the file include/petsclog.h. The source code for the profile logging is in src/sys/plog/.

\subsubsection{Profiling Object Creation and Destruction}

The creation of objects is profiled with the command PetscLogObjectCreate()

PetscLogObjectCreate (PetscObject $\mathrm{h}$ );

which logs the creation of any PETSc object. Just before an object is destroyed, it should be logged with PetscLogObjectDestroy()

PetscLogObjectDestroy (PetscObject h);

These are called automatically by PetscHeaderCreate() and PetscHeaderDestroy(), which are used in creating all objects inherited from the basic object. Thus, these logging routines need never be called directly.

If an object has a clearly defined parent object (for instance, when a work vector is generated for use in a Krylov solver), this information is logged with the command PetscLogObjectParent().

PetscLogObjectParent(PetscObject parent,PetscObject child);

It is also useful to log information about the state of an object, as can be done with the command PetscLogObjectState (PetscObject $\mathrm{h}$, const char *format,...);

For example, for sparse matrices we usually log the matrix dimensions and number of nonzeros.

\subsubsection{Profiling Events}

Events are logged by using the pair

PetscLogEventBegin (PetscLogEvent event, PetscObject o1, .., PetscObject o4);

PetscLogEventEnd (PetscLogEvent event, PetscObject o1, .., PetscObject o4);

This logging is usually done in the abstract interface file for the operations, for example, src/mat/ interface/matrix.c.

\subsubsection{Controlling Profiling}

Routines that control the default profiling available in PETSc include the following

- PetscLogDefaultBegin();

- PetsclogaliBegin();

- PetscLogDump(const char *filename);

- PetscLogView(PetscViewer);

These routines are normally called by the PetscInitialize() and PetscFinalize() routines when the option -log_view is given. 


\section{Chapter 4}

\section{Basic Object Design and Implementation}

PETSc is designed by using strong data encapsulation. Hence, any collection of data (for instance, a sparse matrix) is stored in a way that is completely private from the application code. The application code can manipulate the data only through a well-defined interface, since it does not "know" how the data is stored internally.

\subsection{Introduction}

PETSc is designed around several classes including Vec (vectors) and Mat (matrices, both dense and sparse). Each class is implemented by using a $\mathrm{C}$ struct that contains the data and function pointers for operations on the data (much like virtual functions in $\mathrm{C}++$ classes). Each class consists of three parts:

1. A (small) common part shared by all PETSc classes (for example, both KSP and PC have this same header).

2. Another common part shared by all PETSc implementations of the class (for example, both KSPGMRES and KSPCG have this common subheader).

3. A private part used by only one particular implementation written in PETSc.

For example, all matrix (Mat) classes share a function table of operations that may be performed on the matrix; all PETSc matrix implementations share some additional data fields, including matrix parallel layout, while a particular matrix implementation in PETSc (say compressed sparse row) has its own data fields for storing the actual matrix values and sparsity pattern. This will be explained in more detail in the following sections. New class implementations must use the PETSc common part.

We will use <class $\rangle_{-}<i m p l e m e n t a t i o n>$ to denote the actual source code and data structures used for a particular implementation of an object that has the <class $>$ interface.

\subsection{Organization of the Source Code}

Each class has the following organization.

- Its own, application-public, include file include/petsc<class $>$.h.

- Its own directory, src/<class $>$ or src/<package $>/<$ class $\rangle$. 
- A data structure defined in the file include/petsc/private/<class>impl.h. This data structure is shared by all the different PETSc implementations of the class. For example, for matrices it is shared by dense, sparse, parallel, and sequential formats.

- An abstract interface that defines the application-callable functions for the class. These are defined in the directory $\mathrm{src} /<\mathrm{class}\rangle /$ interface. This is how polymorphism is supported with code that implements the abstract interface to the operations on the object. Essentially, these routines do some error checking of arguments and logging of profiling information and then call the function appropriate for the particular implementation of the object. The name of the abstract function is <class>Operation, for instance, MatMult () or PCCreate (), while the name of a particular implementation is <class>Operation_<implementation>, for instance, MatMult_SeqAIJ() or PCCreate_ILU(). These naming conventions are used to simplify code maintenance (also see Section 2.1).

- One or more actual implementations of the class (for example, sparse uniprocessor and parallel matrices implemented with the AIJ storage format). These are each in a subdirectory of src/<class $>$ impls. Except in rare circumstances, data structures defined here should not be referenced from outside this directory.

Each type of object (for instance, a vector) is defined in its own public include file, by typedef _p_<class>* <class>; (for example, typedef _p_Vec* Vec;). This organization allows the compiler to perform type checking on all subroutine calls while at the same time completely removing the details of the implementation of _p_<class $>$ from the application code. This capability is extremely important because it allows the library internals to be changed without altering or recompiling the application code.

\subsection{Common Object Header}

All PETSc/PETSc objects have the following common header structures defined in include/ petsc/private/petscimpl.h:

Listing 4.1: Function table common to all PETSc-compatible classes

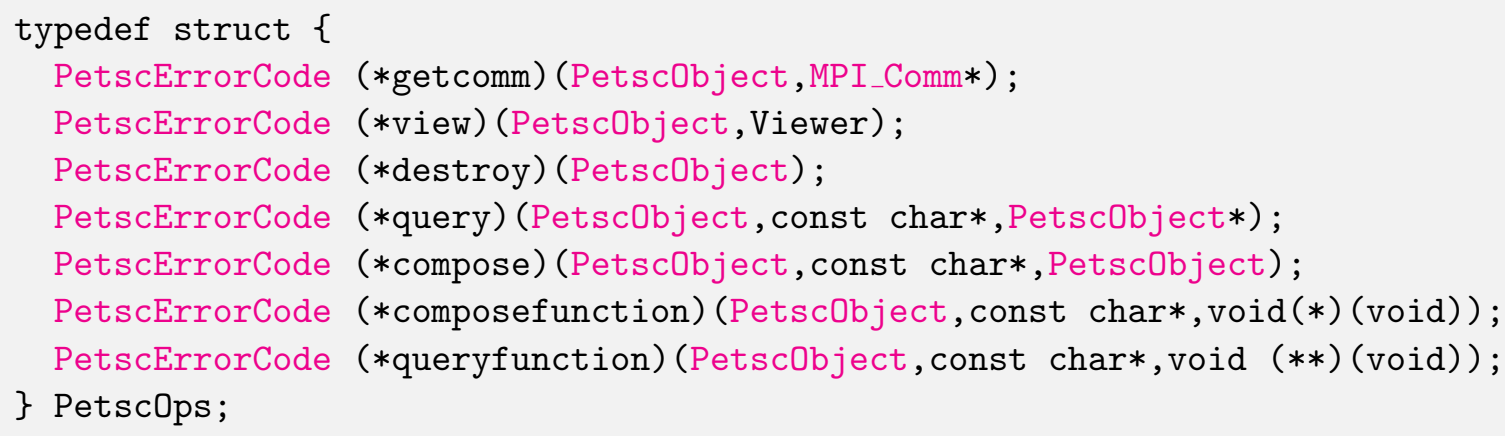

Listing 4.2: Data structure header common to all PETSc-compatible classes

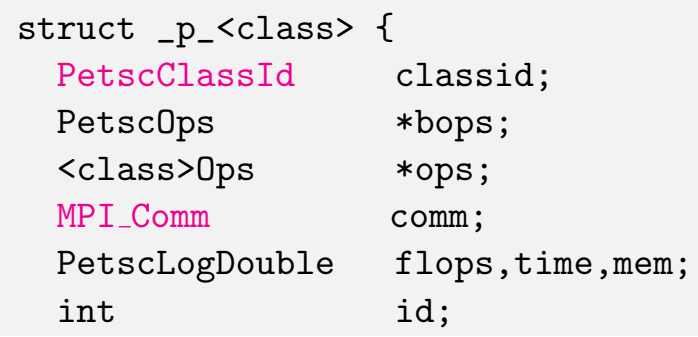




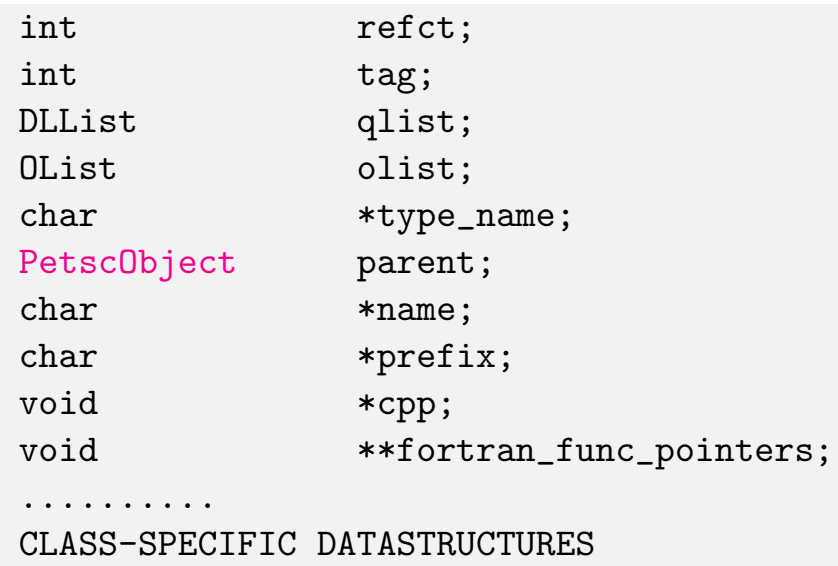

Here <class>ops is a function table (like the PetscOps above) that contains the function pointers for the operations specific to that class. For example, the PETSc vector class object operations in include/petsc/private/vecimpl.h include the following.

Listing 4.3: Function table common to all PETSc-compatible vector objects (truncated)

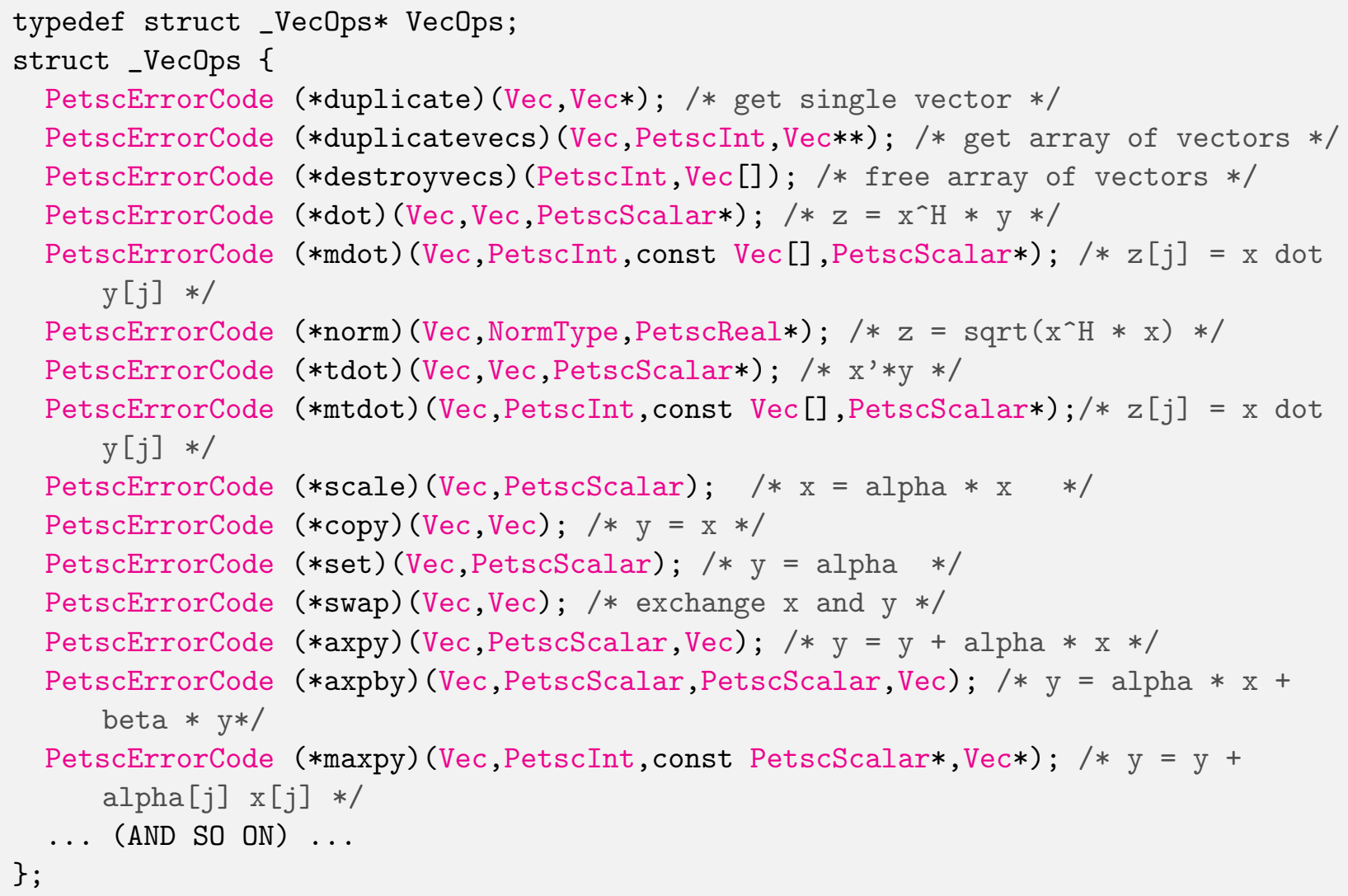

Listing 4.4: Data structure header common to all PETSc vector classes

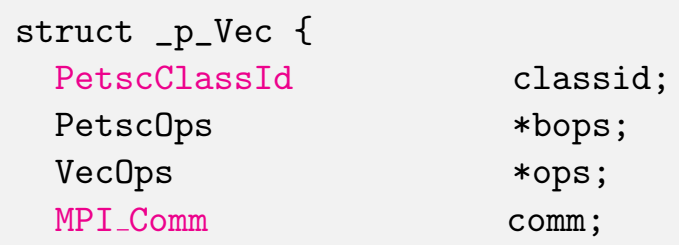




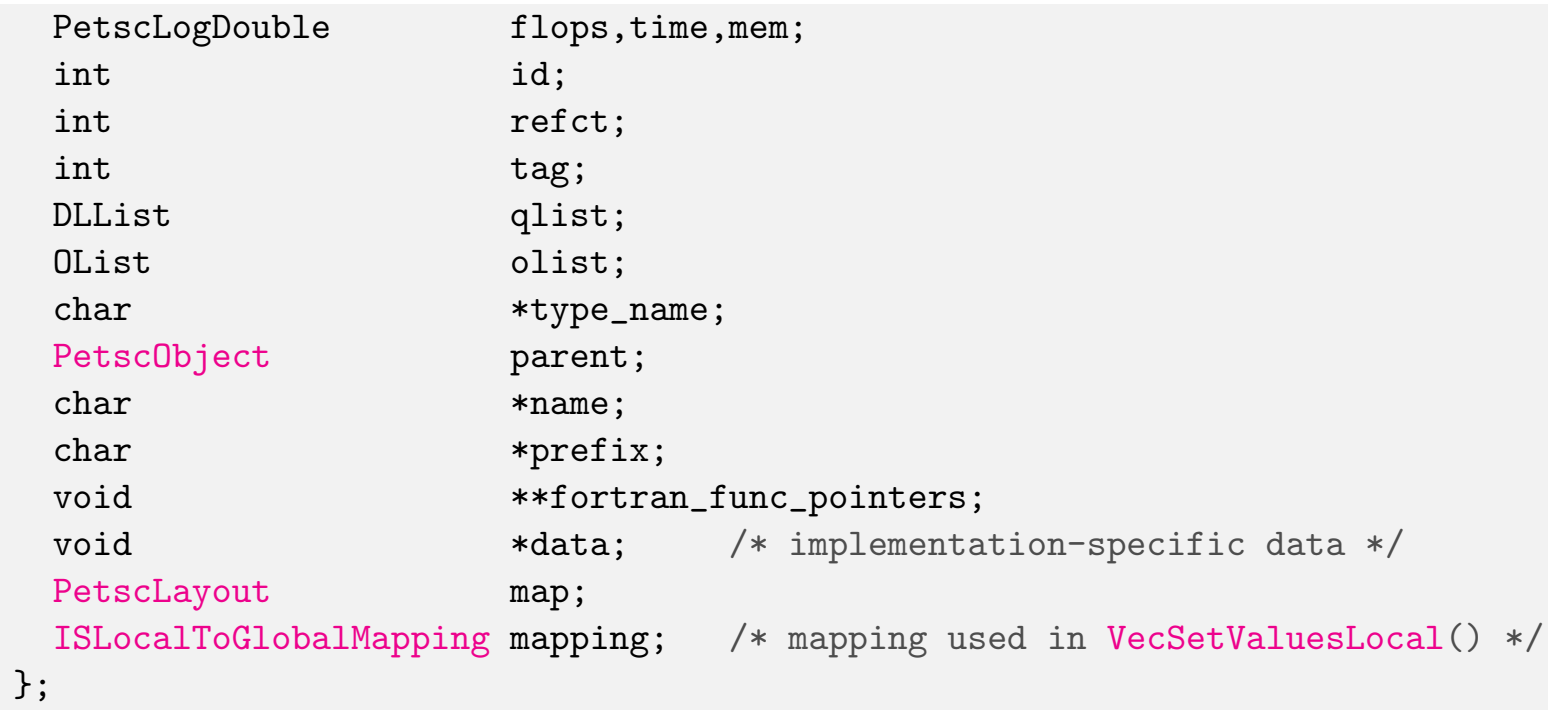

Each PETSc object begins with a PetscClassId, which is used for error checking. Each different class of objects has its value for classid; these are used to distinguish between classes. When a new class is created you need to call

PetscClassIdRegister (const char *classname, PetscClassId *classid);

For example,

PetscClassIdRegister("index set",\&IS_CLASSID);

you can verify that an object is valid of a particular class with PetscValidHeaderSpecific, for example,

PetscValidHeaderSpecific (x,VEC_CLASSID , 1);

The third argument to this macro indicates the position in the calling sequence of the function the object was passed in. This is to generate more complete error messages.

To check for an object of any type, use

PetscValidHeader $(\mathrm{x}, 1)$;

\subsection{Common Object Functions}

Several routines are provided for manipulating data within the header. These include the specific functions in the PETSc common function table. The function pointers are not called directly; rather you should call PetscObjectFunctionName(), where FunctionName is one of the functions listed below with the first letter of each word capitalized.

- getcomm (PetscObject, MPI_Comm*) obtains the MPI communicator associated with this object.

- view(PetscObject, PetscViewer) allows you to store or visualize the data inside an object. If the Viewer is NULL, then it should cause the object to print information on the object to lstinlinestdout.

- destroy(PetscObject) causes the reference count of the object to be decreased by one or the object to be destroyed and all memory used by the object to be freed when the reference 
count drops to zero. If the object has any other objects composed with it, they are each sent a destroy(); that is, the destroy() function is called on them also.

- compose (PetscObject, const char *name,PetscObject) associates the second object with the first object and increases the reference count of the second object. If an object with the same name was previously composed, that object is dereferenced and replaced with the new object. If the second object is NULL and an object with the same name has already been composed, that object is dereferenced (the destroy() function is called on it, and that object is removed from the first object). This is a way to remove, by name, an object that was previously composed.

- query(PetscObject, const char *name,PetscObject*) retrieves an object that was previously composed with the first object via PetscObjectCompose(). It retrieves a NULL if no object with that name was previously composed.

- composefunction(PetscObject, const char *name, void $*$ func) associates a function pointer with an object. If the object already had a composed function with the same name, the old one is replaced. If func is NULL, the existing function is removed from the object. The string name is the character string name of the function.

For example, fname may be PCCreate_LU.

- queryfunction(PetscObject, const char $*$ name, void $* * f u n c)$ retrieves a function pointer that was associated with the object via PetscObjectComposeFunction(). If dynamic libraries are used, the function is loaded into memory at this time (if it has not been previously loaded), not when the composefunction() routine was called.

Since the object composition allows one to compose PETSc objects only with PETSc objects rather than any arbitrary pointer, PETSc provides the convenience object PetscContainer, created with the routine PetscContainerCreate(MPI_Comm,PetscContainer*), to allow wrapping any kind of data into a PETSc object that can then be composed with a PETSc object.

\subsection{Object Function Implementation}

This section discusses how PETSc implements the compose(), query(), composefunction(), and queryfunction() functions for its object implementations. Other PETSc-compatible class implementations are free to manage these functions in any manner; but unless there is a specific reason, they should use the PETSc defaults so that the library writer does not have to "reinvent the wheel."

\subsubsection{Compose and Query Objects}

In src/sys/objects/olist.c, PETSc defines a C struct

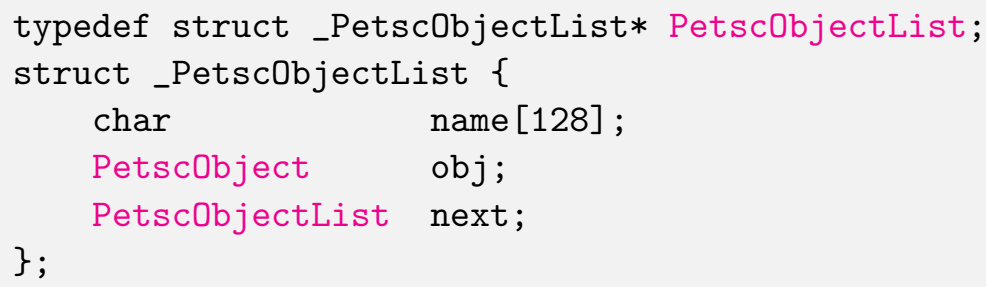

from which linked lists of composed objects may be constructed. The routines to manipulate these elementary objects are

int PetscObjectListAdd(PetscObjectList $* \mathrm{fl}$, const char *name, PetscObject obj);

int PetscObjectListDestroy(PetscObjectList $* f 1)$; 
int PetscObjectListFind(PetscObjectList $f l$, const char *name,PetscObject *obj)

int PetscObjectListDuplicate(PetscObjectList fl,PetscObjectList *nl);

The function PetscObjectListAdd() will create the initial PetscObjectList if the argument $f l$ points to a NULL.

The PETSc object compose() and query() functions are as follows (defined in src/sys/ objects/inherit.c).

PetscErrorCode PetscObjectCompose_Petsc(PetscObject obj, const char

*name, PetscObject ptr)

\{

PetscErrorCode ierr;

PetscFunctionBegin;

PetscObjectListAdd (\&obj->olist, name,ptr);

PetscFunctionReturn( 0$)$;

\}

PetscErrorCode PetscObjectQuery_Petsc(PetscObject obj, const char

*name, PetscObject *ptr)

\{

PetscErrorCode ierr;

PetscFunctionBegin;

PetscObjectListFind (obj->olist, name,ptr);

PetscFunctionReturn( 0$)$;

\}

\subsubsection{Compose and Query Functions}

PETSc allows you to compose functions by specifying a name and function pointer. In src/sys/ $\mathrm{dll} / \mathrm{reg}$.c, PETSc defines the following linked list structure.

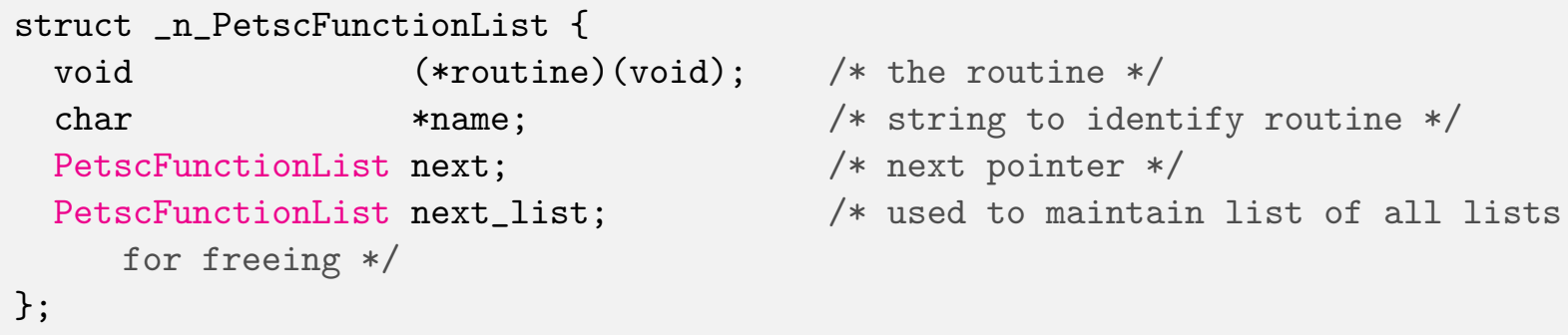

Each PETSc object contains a PetscFunctionList object. The composefunction() and queryfunction() are given by the following.

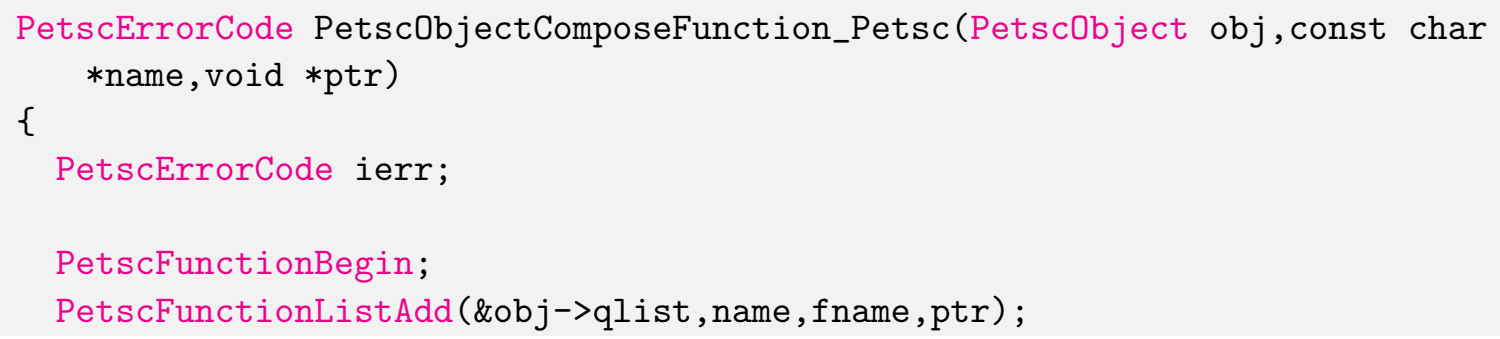




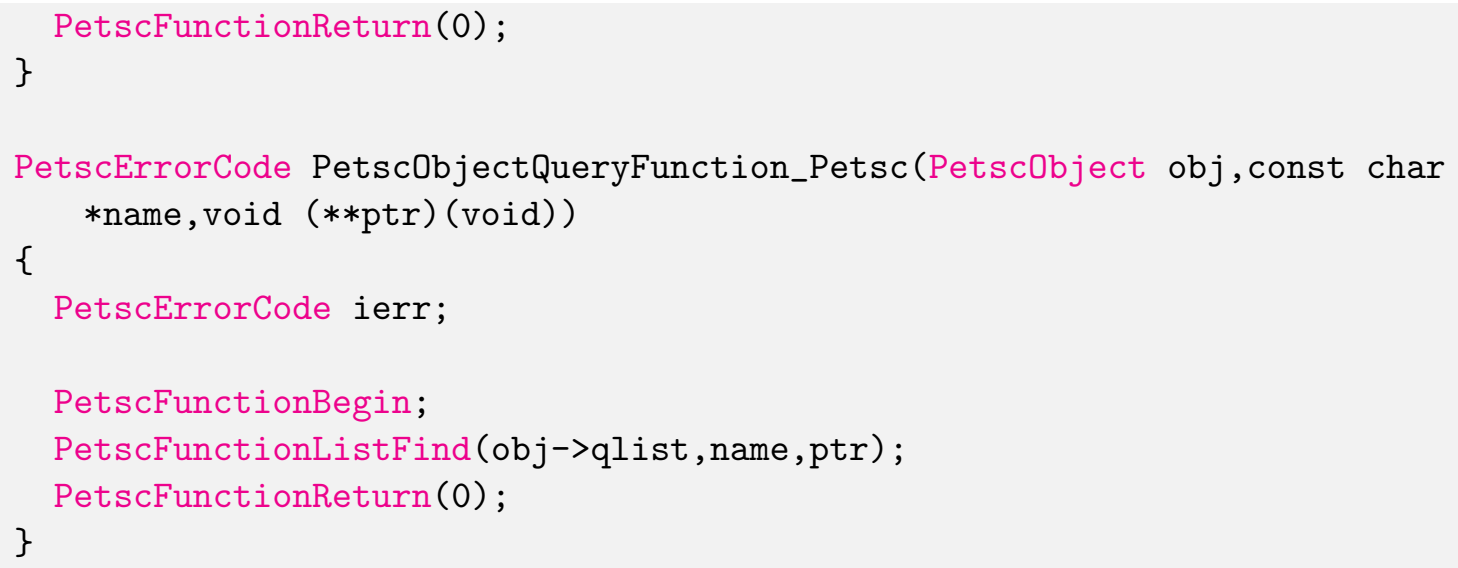

In addition to using the PetscFunctionList mechanism to compose functions into PETSc objects, it is also used to allow registration of new class implementations; for example, new preconditioners.

\subsubsection{Simple PETSc Objects}

Some simple PETSc objects do not need PETSCHEADER and the associated functionality. These objects are internally named as _n_ $\mathrm{n}_{-}$class $\rangle$as opposed to _p_<class $\rangle$, for example, _n_PetscTable vs _p_Vec.

\subsection{PETSc Packages}

The PETSc source code is divided into the following library-level packages: sys, Vec, Mat, DM, KSP, SNES, TS, TAO. Each of these has a directory under the src directory in the PETSc tree and, optionally, can be compiled into separate libraries. Each package defines one or more classes; for example, the KSP package defines the KSP and PC classes, as well as several utility classes. In addition, each library-level package may contain several class-level packages associated with individual classes in the library-level package. In general, most "important" classes in PETSc have their own class level package. Each package provides a registration function XXXInitializePackage(), for example KSPInitializePackage(), which registers all the classes and events for that package. Each package also registers a finalization routine, XXXFinalizePackage(), that releases all the resources used in registering the package, using PetscRegisterFinalize(). The registration for each package is performed "on demand" the first time a class in the package is utilized. This is handled, for example, with code such as

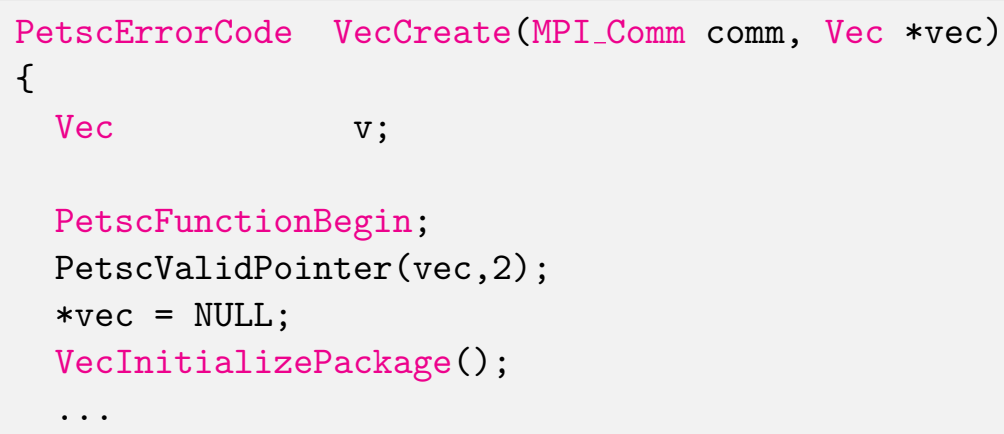




\section{Chapter 5}

\section{How the Solvers Handle User Provided Callbacks}

The solver objects in PETSc, KSP (optionally), SNES, and TS require user provided callback functions (and contexts for the functions) that define the problem to be solved. These functions are supplied by the user with calls such as SNESSetFunction(SNES,...) and TSSetRHSFunction(TS,...). One would naturally think that the functions provided would be attached to the appropriate solver object, that is, that the SNES callbacks would be attached to the SNES object and TS callbacks to the TS object. This is not the case. Or possibly one might think the callbacks would be attached to the DM object associated with the solver object. This is also not the case. Rather, the callback functions are attached to an inner nonpublic DMXXX object (XXX is KSP, SNES, or TS) that is attached to the DM that is attached to the XXX solver object. This convoluted design is to support multilevel and multidomain solvers where different levels and different domains may (or may not) share the same callback function or callback context. You can control exactly what XXX/DM objects share a common DMXXX object.

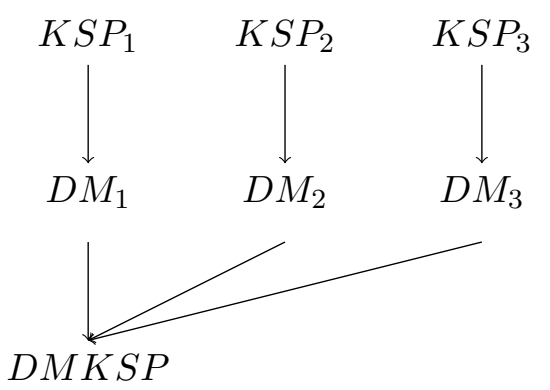

Figure 1: Three levels of KSP/DM share the same DMKSP

In Figure 1 we depict how three levels of KSP objects share a common DMKSP object. The code to access the inner DMKSP object is

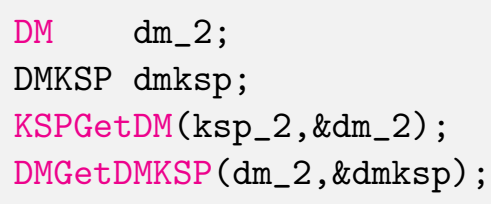

To obtain a new DMKSP object for which you can change the callback functions (or their contexts) without affecting the original DMKSP, call 


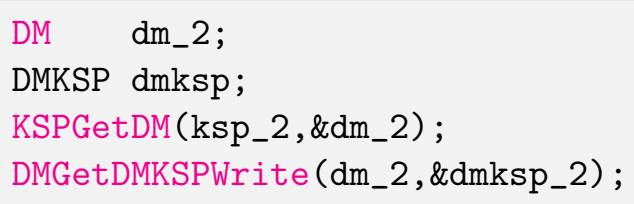

This results in the object organization as indicated in Figure 2

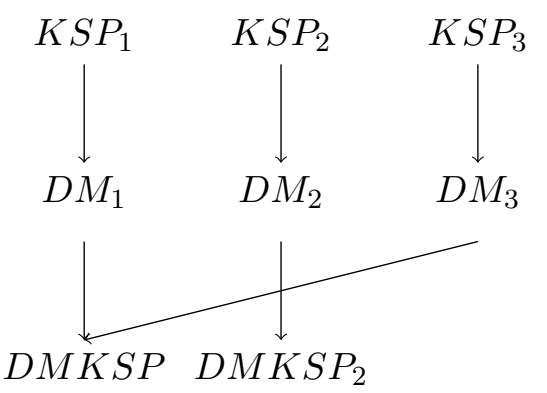

Figure 2: Two levels of KSP/DM share the same DMKSP; one has its own private copy

The DMKSP object is essentially the list of callback functions and their contexts, for example,

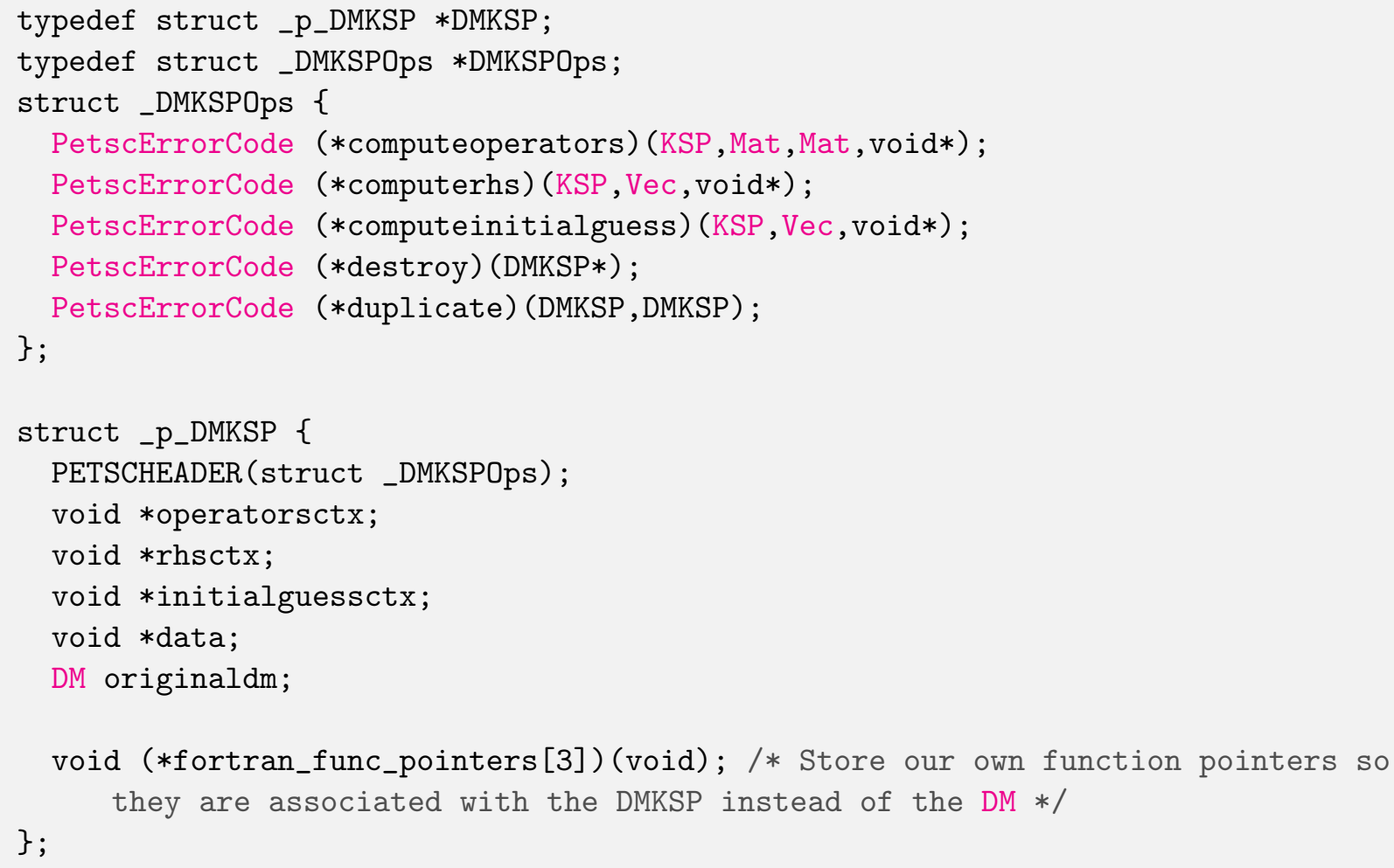

We now explore in more detail exactly how the solver calls set by the user are passed down to the inner DMKSP object. For each user level solver routine for setting a callback a similar routine exists at the DM level. Thus, XXXSetY $(\mathrm{XXX}, \ldots)$ has a routine DMXXXSetY (DM, ...).

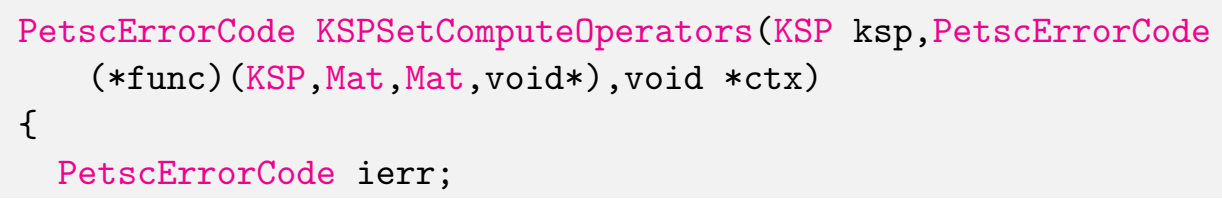




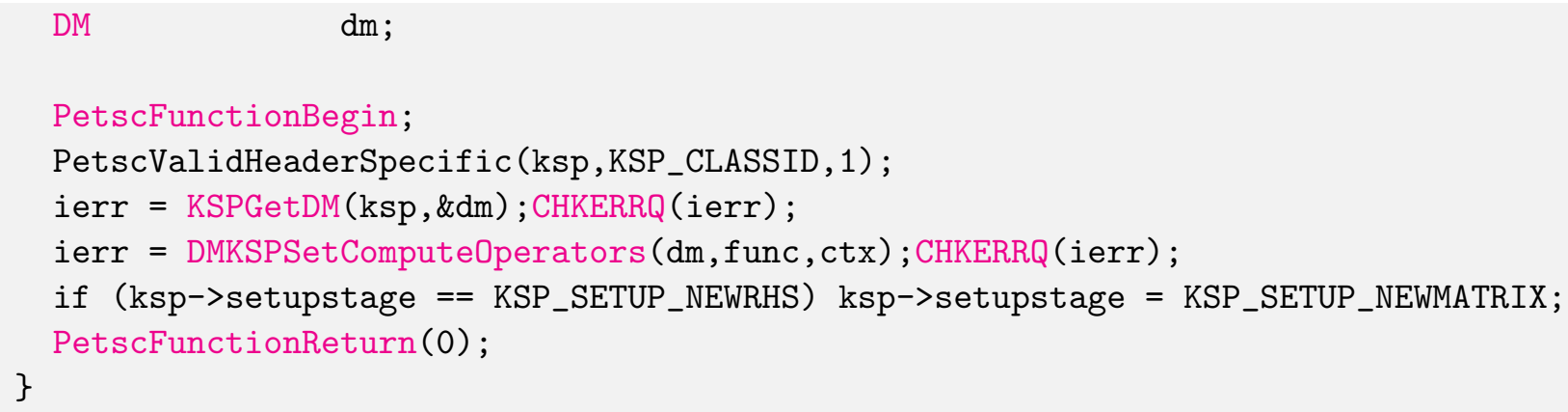

The implementation of DMXXXSetY $(D M, \ldots)$ gets a "writable" version of the DMXXX object via DMGetDMXXXWrite (DM,DMXXX*) and sets the function callback and its context into the DMXXX object.

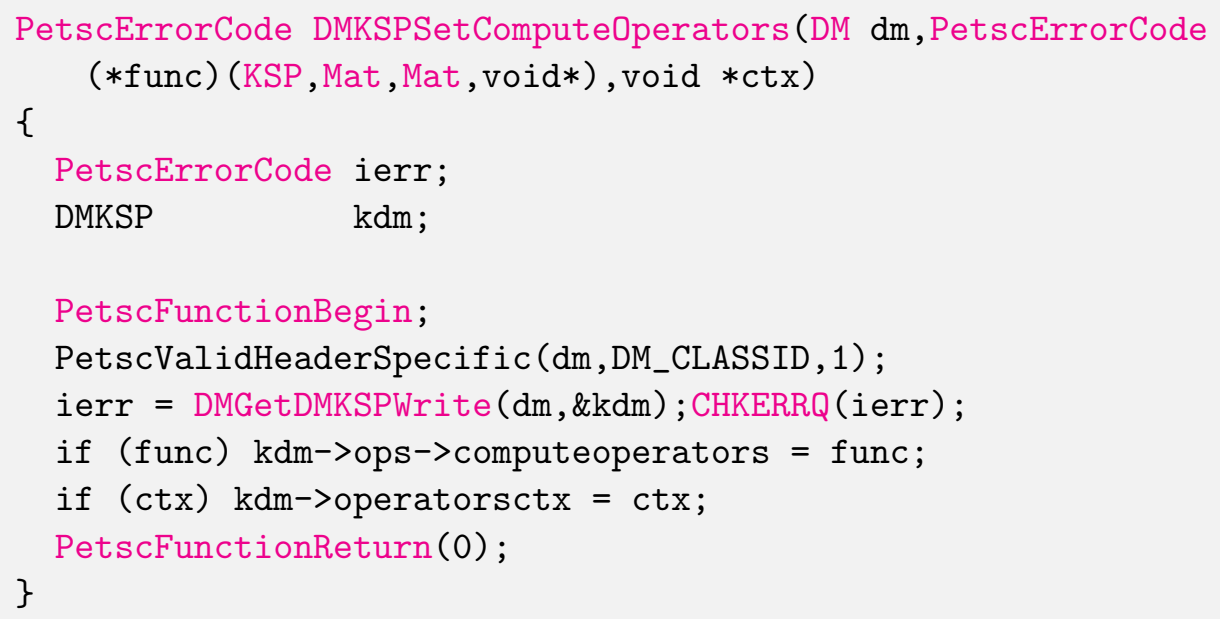

The routine for DMGetDMXXXWrite (DM,DMXXX*) entails a duplication of the object unless the DM associated with the DMXXX object is the original DM that the DMXXX object was created with. This can be seen in the following code.

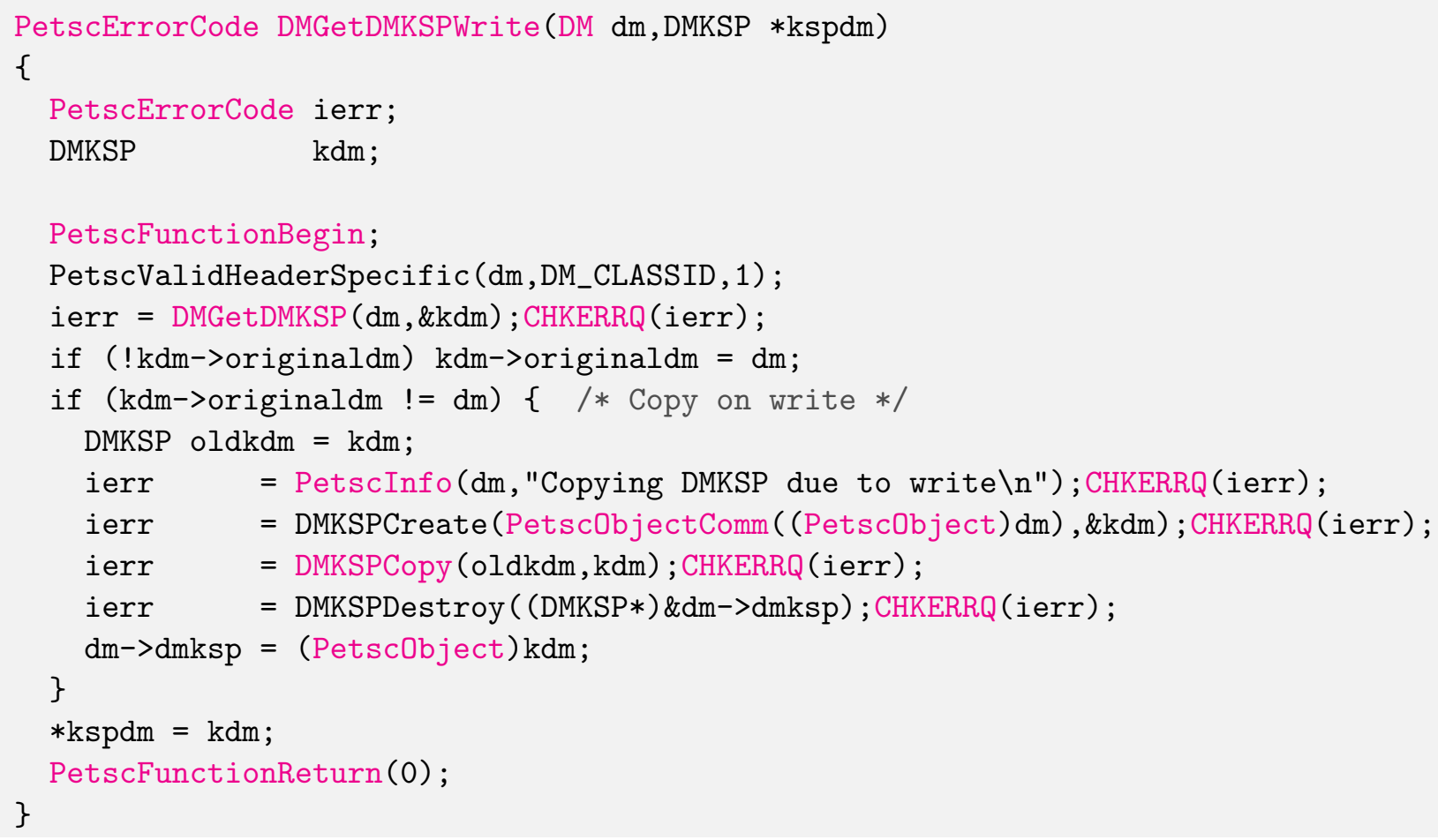


The routine DMGetDMXXX (DM, DMXXX*) has the following form.

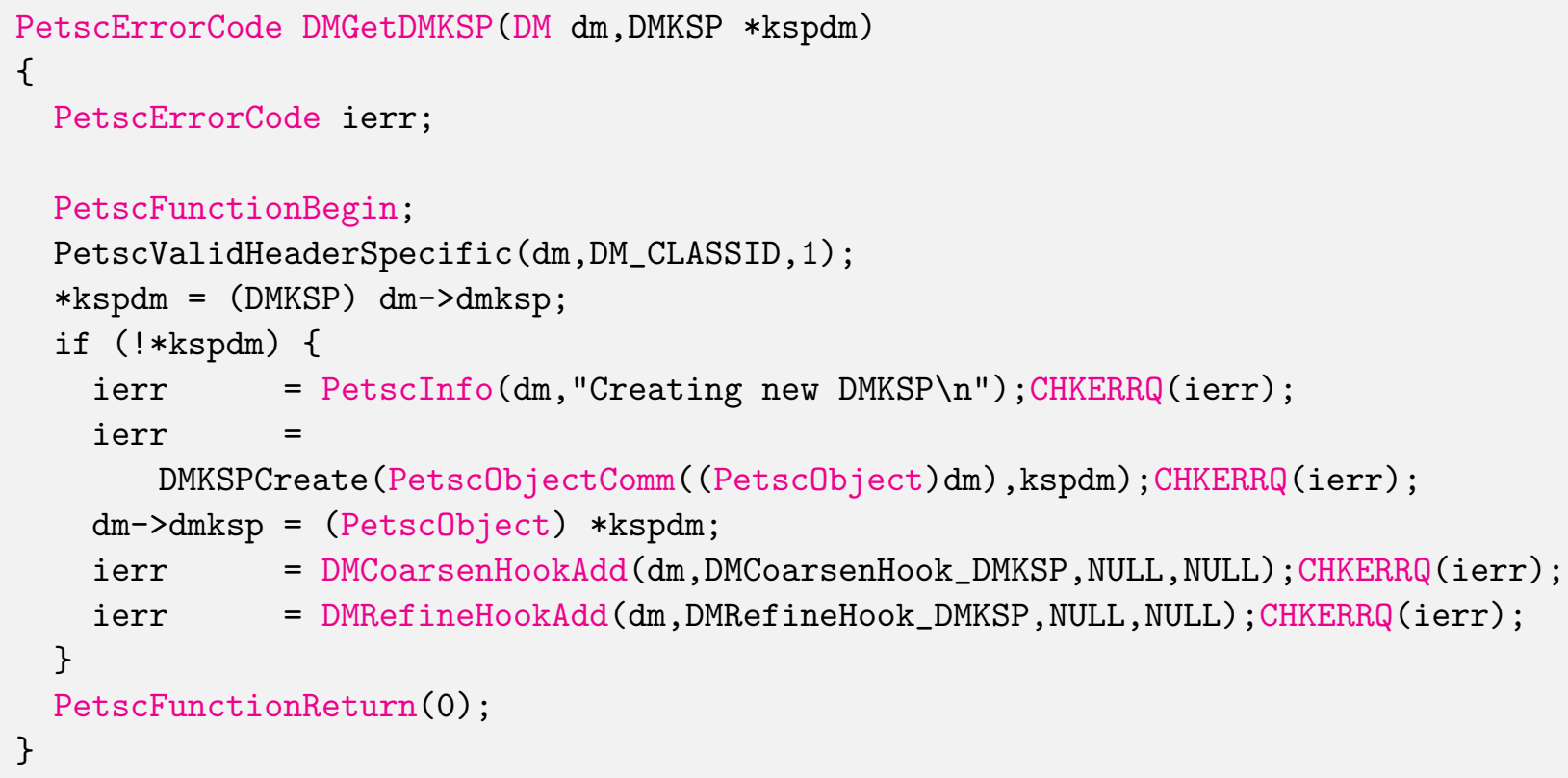

This routine uses DMCoarsenHookAdd() and DMCoarsenHookAdd() to attach to the DM object two functions that are automatically called when the object is coarsened or refined. The hooks DMCoarsenHook_DMXXX() and DMCoarsenHook_DMXXX() have the form.

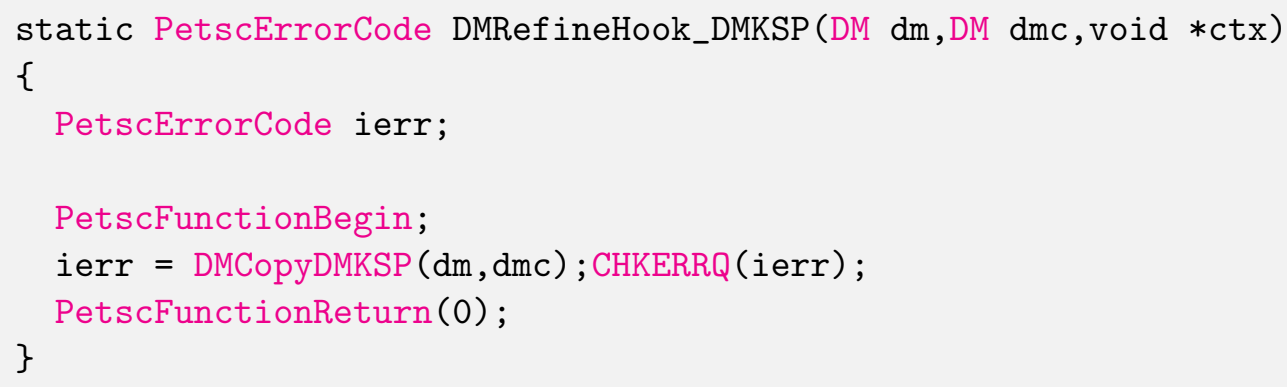

where

PetscErrorCode DMCopyDMKSP (DM dmsrc,DM dmdest)

\{

PetscErrorCode ierr;

PetscFunctionBegin;

PetscValidHeaderSpecific(dmsrc,DM_CLASSID , 1);

PetscValidHeaderSpecific (dmdest, DM_CLASSID , 2);

ierr $\quad=$ DMKSPDestroy ( (DMKSP $*)$ \&dmdest->dmksp);CHKERRQ (ierr);

dmdest->dmksp $=$ dmsrc->dmksp;

ierr $\quad=$ PetscObjectReference (dmdest->dmksp);CHKERRQ(ierr);

ierr $\quad=$

DMCoarsenHookAdd (dmdest, DMCoarsenHook_DMKSP , NULL , NULL) ; CHKERRQ (ierr); ierr =

DMRef ineHookAdd (dmdest, DMRef ineHook_DMKSP, NULL , NULL) ; CHKERRQ (ierr) ; PetscFunctionReturn(0); 
\}

ensures that the new DM shares the same DMXXX as the parent DM and also inherits the hooks if it is refined or coarsened.

If you provide callbacks to a solver after the DM associated with a solver has been refined or coarsened, those child DMs will not share a common DMXXX.

The TS object manages its callback functions in a way similar to KSP and SNES, although there are no multilevel TS implementations so in theory the DMTS object is currently unneeded. 


\section{Chapter 6}

\section{The Various Matrix Classes}

PETSc provides a variety of matrix implementations, since no single matrix format is appropriate for all problems. This section first discusses various matrix blocking strategies and then describes the assortment of matrix types in PETSc.

\subsection{Matrix Blocking Strategies}

In today's computers, the time to perform an arithmetic operation is dominated by the time to move the data into position, not the time to compute the arithmetic result. For example, the time to perform a multiplication operation may be one clock cycle, while the time to move the floating-point number from memory to the arithmetic unit may take 10 or more cycles. In order to help manage this difference in time scales, most processors have at least three levels of memory: registers, cache, and random access memory. (In addition, some processors have external caches, and the complications of paging introduce another level to the hierarchy.)

Thus, to achieve high performance, a code should first move data into cache and from there move it into registers and use it repeatedly while it remains in the cache or registers before returning it to main memory. If a floating-point number is reused 50 times while it is in registers, then the "hit" of 10 clock cycles to bring it into the register is not important. But if the floating-point number is used only once, the "hit" of 10 clock cycles becomes noticeable, resulting in disappointing flop rates.

Unfortunately, the compiler controls the use of the registers, and the hardware controls the use of the cache. Since the user has essentially no direct control, code must be written in such a way that the compiler and hardware cache system can perform well. Good-quality code is then said to respect the memory hierarchy.

The standard approach to improving the hardware utilization is to use blocking. That is, rather than working with individual elements in the matrices, you employ blocks of elements. Since the use of implicit methods in PDE-based simulations leads to matrices with a naturally blocked structure (with a block size equal to the number of degrees of freedom per cell), blocking is advantageous. The PETSc sparse matrix representations use a variety of techniques for blocking, including the following:

- Storing the matrices using a generic sparse matrix format, but storing additional information about adjacent rows with identical nonzero structure (so-called I-nodes); this I-node information is used in the key computational routines to improve performance (the default for the MATSEQAIJ and MATMPIAIJ formats). 
- Storing the matrices using a fixed (problem dependent) block size (via the MATSEQBAIJ and MATMPIBAIJ formats).

The advantage of the first approach is that it is a minimal change from a standard sparse matrix format and brings a large percentage of the improvement obtained via blocking. Using a fixed block size gives the best performance, since the code can be hardwired with that particular size (for example, in some problems the size may be 3 , in others 5 , and so on), so that the compiler will then optimize for that size, removing the overhead of small loops entirely.

The following table presents the floating-point performance for a basic matrix-vector product using three approaches: a basic compressed row storage format (using the PETSc runtime options -mat_seqaij -mat_no_unroll); the same compressed row format using I-nodes (with the option -mat_seqaij); and a fixed block size code, with a block size of 3 for these problems (using the option -mat_seqbaij). The rates were computed on one node of an older IBM Power processsor based system, using two test matrices. The first matrix (ARCO1), courtesy of Rick Dean of Arco, arises in multiphase flow simulation; it has 1,501 degrees of freedom, 26,131 matrix nonzeros, a natural block size of 3 , and a small number of well terms. The second matrix (CFD), arises in a three-dimensional Euler flow simulation and has 15,360 degrees of freedom, 496,000 nonzeros, and a natural block size of 5 . In addition to displaying the flop rates for matrix-vector products, we display them for triangular solves obtained from an ILU(0) factorization.

\begin{tabular}{|c|c|c|c|c|}
\hline Problem & Block size & Basic & I-node version & Fixed block size \\
\hline \multicolumn{5}{|c|}{ Matrix-Vector Product (Mflop/sec) } \\
\hline Multiphase & 3 & 27 & 43 & 70 \\
\hline Euler & 5 & 28 & 58 & 90 \\
\hline \multicolumn{5}{|c|}{ Triangular Solves from ILU(0) (Mflop/sec) } \\
\hline Multiphase & 3 & 22 & 31 & 49 \\
\hline Euler & 5 & 22 & 39 & 65 \\
\hline
\end{tabular}

These examples demonstrate that careful implementations of the basic sequential kernels in PETSc can dramatically improve overall floating point performance, and users can immediately benefit from such enhancements without altering a single line of their application codes. Note that the speeds of the I-node and fixed block operations are several times that of the basic sparse implementations.

\subsection{Assorted Matrix Types}

PETSc offers a variety of both sparse and dense matrix types.

\subsubsection{Sequential AIJ Sparse Matrices}

The default matrix representation within PETSc is the general sparse AIJ format (also called the Yale sparse matrix format or compressed sparse row format, CSR).

\subsubsection{Parallel AIJ Sparse Matrices}

The AIJ sparse matrix type, is the default parallel matrix format; additional implementation details are given in [4]. 


\subsubsection{Sequential Block AIJ Sparse Matrices}

The sequential and parallel block AIJ formats, which are extensions of the AIJ formats described above, are intended especially for use with multiclass PDEs. The block variants store matrix elements by fixed-sized dense $\mathrm{nb} \times \mathrm{nb}$ blocks. The stored row and column indices begin at zero.

The routine for creating a sequential block AIJ matrix with m rows, $\mathrm{n}$ columns, and a block size of $\mathrm{nb}$ is

MatCreateSeqBAIJ(MPI_Comm comm, int $\mathrm{nb}$, int $\mathrm{m}$, int $\mathrm{n}$, int $\mathrm{nz}$, int $* \mathrm{nnz}$, Mat $* \mathrm{~A}$ )

The arguments $\mathrm{nz}$ and $\mathrm{nnz}$ can be used to preallocate matrix memory by indicating the number of block nonzeros per row. For good performance during matrix assembly, preallocation is crucial; however, you can set $\mathrm{nz}=0$ and $\mathrm{nnz}=\mathrm{NULL}$ for PETSc to dynamically allocate matrix memory as needed. The PETSc users manual discusses preallocation for the AIJ format; extension to the block AIJ format is straightforward.

Note that the routine MatSetValuesBlocked() can be used for more efficient matrix assembly when using the block AIJ format.

\subsubsection{Parallel Block AIJ Sparse Matrices}

Parallel block AIJ matrices with block size nb can be created with the command MatCreateBAIJ()

MatCreateBAIJ(MPIComm comm, int $\mathrm{nb}$, int $\mathrm{m}$, int $\mathrm{n}$, int $\mathrm{M}$, int $\mathrm{N}$, int $\mathrm{d}$, nz, int

*d_nnz, int o_nz, int *o_nnz, Mat *A);

A is the newly created matrix, while the arguments $\mathrm{m}, \mathrm{n}, \mathrm{M}$, and $\mathrm{N}$ indicate the number of local rows and columns and the number of global rows and columns, respectively. Either the local or global parameters can be replaced with PETSC_DECIDE, so that PETSc will determine them. The matrix is stored with a fixed number of rows on each processor, given by $\mathrm{m}$, or determined by PETSc if $\mathrm{m}$ is PETSC_DECIDE.

If PETSC_DECIDE is not used for $\mathrm{m}$ and $\mathrm{n}$ then you must ensure that they are chosen to be compatible with the vectors. To do so, you first consider the product $y=A x$. The $\mathrm{m}$ that used in MatCreateBAIJ() must match the local size used in the VecCreateMPI() for y. The $\mathrm{n}$ used must match that used as the local size in VecCreateMPI() for $\mathrm{x}$.

You must set d_nz=0, o_nz=0, d_nnz=NULL, and o_nnz=NULL for PETSc to control dynamic allocation of matrix memory space. Analogous to $\mathrm{nz}$ and $\mathrm{nnz}$ for the routine MatCreateSeqBAIJ(), these arguments optionally specify block nonzero information for the diagonal (d_nz and d_nnz) and off-diagonal (o_nz and o_nnz) parts of the matrix. For a square global matrix, we define each processor's diagonal portion to be its local rows and the corresponding columns (a square submatrix); each processor's off-diagonal portion encompasses the remainder of the local matrix (a rectangular submatrix). The PETSc users manual gives an example of preallocation for the parallel AIJ matrix format; extension to the block parallel AIJ case is straightforward.

\subsubsection{Sequential Dense Matrices}

PETSc provides both sequential and parallel dense matrix formats, where each processor stores its entries in a column-major array in the usual Fortran style.

\subsubsection{Parallel Dense Matrices}

The parallel dense matrices are partitioned by rows across the processors, so that each local rectangular submatrix is stored in the dense format described above. 


\section{Chapter 7}

\section{PETSc Testing System}

The PETSc test system consists of

1. A language contained within the example source files that describes the tests to be run

2. The test generator (config/gmakegentest.py) that at the make step parses the example source files and generates the makefiles and shell scripts.

3. The petsc test harness that consists of makefile and shell scripts that runs the executables with several logging and reporting features.

Details on using the harness may be found in the main PETSc manual.

\subsection{PETSc Test Description Language}

PETSc tests and tutorials contain within their file a simple language to describe tests and subtests required to run executables associated with compilation of that file. The general skeleton of the file is

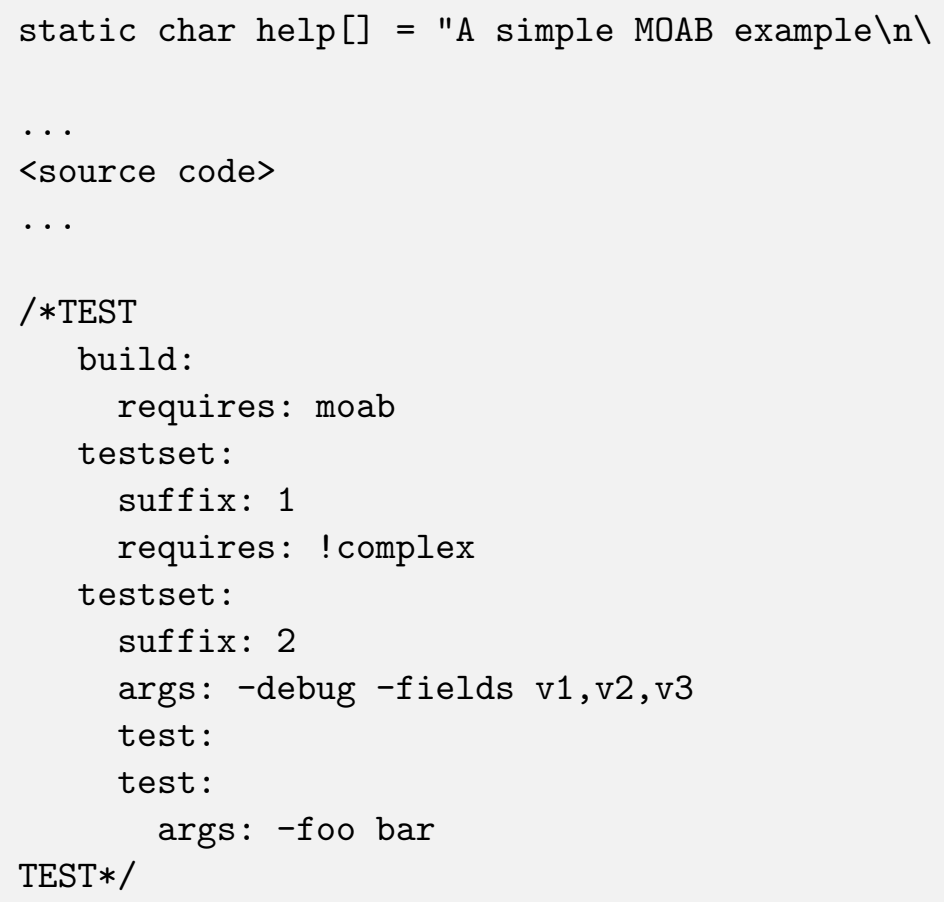


For our language, a test is associated with the following

- A single shell script

- A single makefile

- A single output file that represents the expected esults

- Two or more command tests, usually, one or more mpiexec tests that run the executable, and one or more diff tests to compare output with the expected result.

Our language also supports a testset that specifies either a new test entirely or multiple executable/diff tests within a single test. At the core, the executable/diff test combination will look something like this:

mpiexec $-n$ 1 ..ex1 1> ex1.tmp 2> ex1.err

diff ex1.tmp output/ex1.out 1> diff-ex1.tmp 2> diff-ex1.err

In practice, we want to do various logging and counting by the test harness; as are explained further below. The input language supports simple yet flexible test control, and we begin by describing this language.

\subsubsection{Runtime Language Options}

At the end of each test file, a marked comment block, using YAML, is inserted to describe the test(s) to be run. The elements of the test are done with a set of supported key words that sets up the test.

The goals of the language are to be

1. as minimal as possible with the simplest test requiring only one keyword,

2. independent of the filename such that a file can be renamed without rewriting the tests, and

3. intuitive.

In order to enable the second goal, the basestring of the filename is defined as the filename without the extension; for example, if the filename is ex1.c, then basestring=ex1.

With this background, these keywords are as follows.

testset or test: (Required)

At the top level either a single test or a test set must be specified. All other keywords are sub-entries of this keyword.

suffix: (Optional; Default: suffix="")

- The test name is given by testname = basestring if the suffix is set to an empty string, and by testname = basestring + "_" + suffix otherwise.

- This can be specified only for top level test nodes.

output_file: (Optional; Default: output_file = "output/" + testname + ".out")

- The output of the test is to be compared with an expected result whose name is given by output_file. 
- This file is described relative to the source directory of the source file and should be in the output subdirectory (for example, output/ex1.out)

nsize: (Optional; Default: nsize=1)

- This integer is passed to mpiexec; i.e., mpiexec -n nsize

args: (Optional; Default: " ")

- These arguments are passed to the executable.

TODO: (Optional; Default: False)

- Setting this Boolean to True will tell the test to appear in the test harness but report only TODO per the TAP standard.

- A runscript will be generated and can easily be modified by hand to run.

filter: (Optional; Default: " ")

- Sometimes only a subset of the output is meant to be tested against the expected result. If this keyword is used, it processes the executable output and puts it into the file to be actually compared with output_file.

- The value of this is the command to be run, for example, grep foo or sort -nr.

- If the filter begins with Error:, then the test is assumed to be testing the stderr output, and the error code and output are set up to be tested.

filter_output: (Optional; Default: " ")

- Sometimes filtering the output file is useful for standardizing tests. For example, in order to handle the issues related to parallel output, both the output from the test example and the output file need to be sorted (since sort does not produce the same output on all machines). This works the same as filter to implement this feature

localrunfiles: (Optional; Default: " ")

- The tests are run under PETSC_ARCH/tests, but some tests require runtime files that are maintained in the source tree. Files in this (space-delimited) list will be copied over. If you list a directory instead of files, it will copy the entire directory (this is limited currently to a single directory)

- The copying is done by the test generator and not by creating makefile dependencies.

requires: (Optional; Default: " ")

- This is a space-delimited list of run requirements (not build requirements; see Build requirements below).

- In general, the language supports and and not constructs using ! => not and , $\Rightarrow>$ and.

- MPIUNI should work for all -n 1 examples so this need not be in the requirements list.

- Inputs sometimes require external matrices that are found in the DATAFILES path. For these tests requires: datafilespath can be specifed.

- Packages are indicated with lower-case specification, for example, requires: superlu_dist. 
- Any defined variable in petscconf.h can be specified with the defined(...) syntax, for example, defined (PETSC_USE_INFO).

- Any definition of the form PETSC_HAVE_FOO can just use requires: foo similar to how third-party packages are handled.

timeoutfactor: (Optional; Default: "1")

- This parameter allows you to extend the default timeout for an individual test such that the new timeout time is timeout=(default timeout) $\mathrm{x}$ (timeoutfactor).

- Tests are limited to a set time that is found at the top of "config/petsc_harness.sh" and can be overwritten by passing in the TIMEOUT argument to gmakefile (see make $-f$ gmakefile help.

\subsubsection{Additional Specifications}

In addition to the above keywords, other language features are supported.

- for loops: Specifying $\{\{\ldots\}$ shared output $\}$ or $\{\{\ldots\}$ separate output $\}$ will create for loops over an enclosed space-delmited list. If the loop causes a different output, then separate output would be used. If the loop does not cause separate output, then the shared (in shorthand notation, $\{\{\ldots\}\}$ ) syntax must be used.

For loops are supported within nsize and args. An example is

args: -matload_block_size $\left.\left\{\begin{array}{ll}2 & 3\end{array}\right\}\right\}$

In this case, two execution lines would be addded with two different arguments. Associated diff lines would be added as well automatically.

Here the output for each matload_block_size is assumed to give the same output so that only one diff file is needed. If the variables produced different output, then the separate output option would be added. In this case, each loop variable and value become a separate script.

See examples below for how it works in practice.

\subsubsection{Test Block Examples}

The following is the simplest test block:

/*TESTS

test:

TESTS*/

which is equivalent to

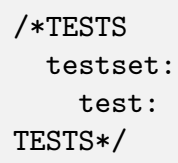

which is equivalent to

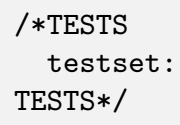


If this block is in src/a/b/examples/tutorials/ex1.c, then it will create a_b_tutorials-ex1 test that requires only one processor/thread, with no arguments, and diff the resultant output with src/a/b/examples/tutorials/output/ex1.out.

For Fortran, the equivalent is

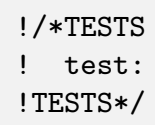

A more complete example is

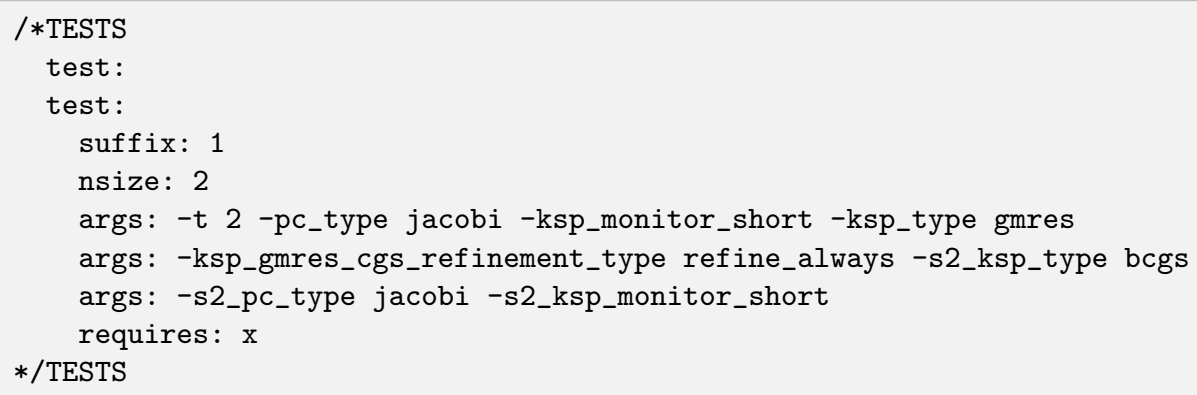

This creates two tests. Assuming that this is src/a/b/examples/tutorials/ex1.c, the tests would be a_b_tutorials-ex1 and a_b_tutorials-ex1_1. file:

Following is an example of how to test a permutuation of arguments against the same output

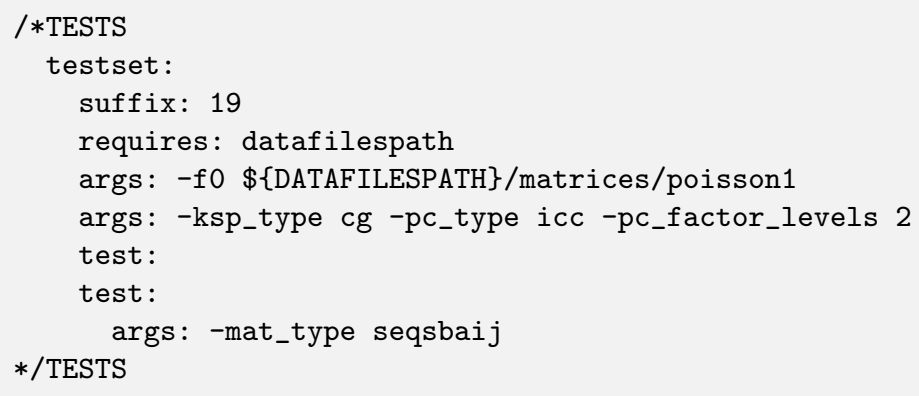

Assuming that this is ex10.c, there would be two mpiexec/diff invocations in runex10_19.sh. Here is a similar example, but the permutation of arguments creates different output:

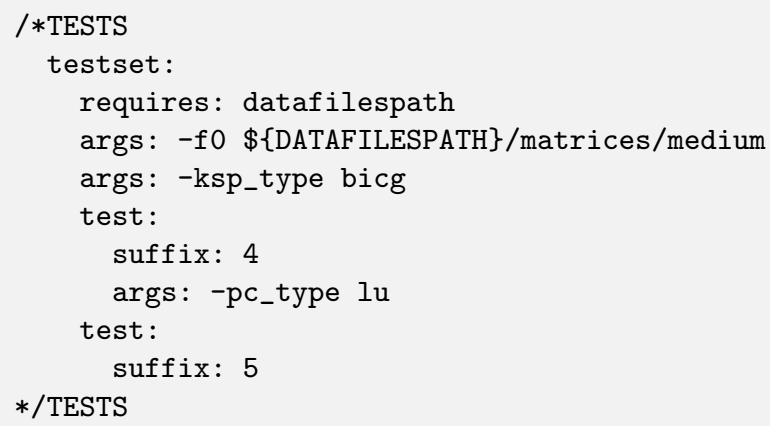

Assuming that this is ex10.c, two shell scripts will be created: runex10_4.sh and runex10_5.sh. An example using a for loop is:

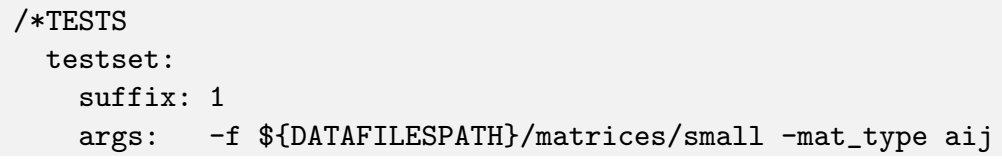




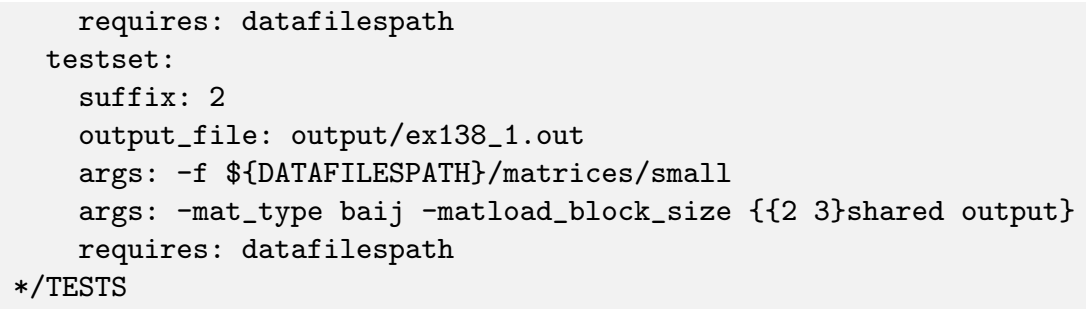

In this example, ex138_2 will invoke runex138_2.sh twice with two different arguments, but both are diffed with the same file.

Following is an example showing the hierarchical nature of the test specification.

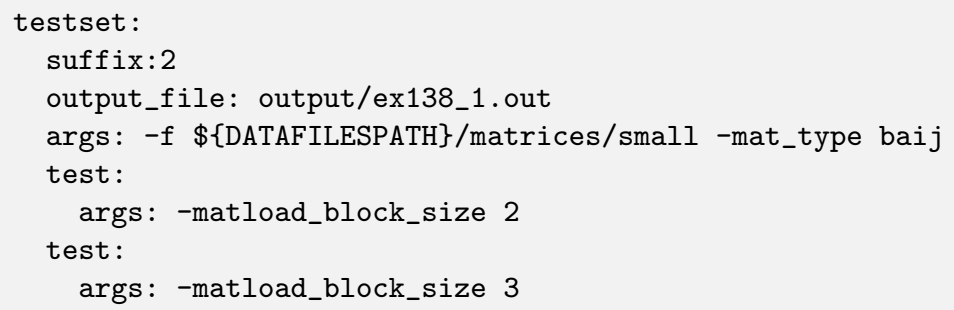

This is functionally equivalent to the for loop shown above.

Here is a more complex example using for loops:

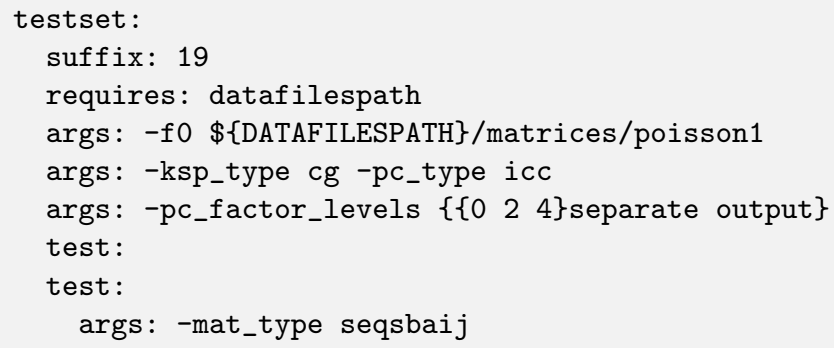

If this is in ex10.c, then the shell scripts generated would be

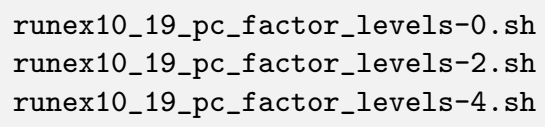

Each shell script would invoke mpiexec twice.

\subsubsection{Build Language Options}

You can specify issues related to the compilation of the source file with the build: block. The language is as follows.

- requires: (Optional; Default: "")

- Same as the runtime requirements (for example, can include requires: fftw) but also requirements related to types:

1. Precision types: single, double, quad, int32

2. Scalar types: complex (and ! complex)

- In addition, TODO is available to allow you to skip the build of this file but still maintain it in the source tree.

- depends: (Optional; Default: " ") 
- List any dependencies required to compile the file

A typical example for compiling for only real numbers is

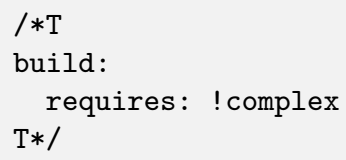

\subsection{PETSC Test Harness}

The goals of the PETSc test harness are threefold.

- Provide standard output used by other testing tools

- Be as lightweight as possible and easily fit within the PETSc build chain

- Provide information on all tests, even those that are not built or run because they do not meet the configuration requirements

Before understanding the test harness, you should first understand the desired requirements for reporting and logging.

\section{Testing the Parsing}

After inserting the language into the file, you can test the parsing by executuing

\$\{PETSC_DIR\}/lib/petsc/bin/maint/testparse.py -t <test src file>

A dictionary will be pretty-printed. From this dictionary printout, any problems in the parsing are is usually obvious. This python file is used by

\$\{PETSC_DIR\}/config/gmakegentest.py

in generating the test harness.

\subsection{Test Output Standards: TAP}

The PETSc test system is designed to be compliant with the Test Anything Protocal (TAP); see https://testanything.org/tap-specification.html

This is a simple standard designed to allow testing tools to work together easily. There are libraries to enable the output to be used easily, including sharness, which is used by the git team. However, the simplicity of the PETSc tests and TAP specification means that we use our own simple harness given by a single shell script that each file sources: petsc_harness.sh.

As an example, consider this test input:

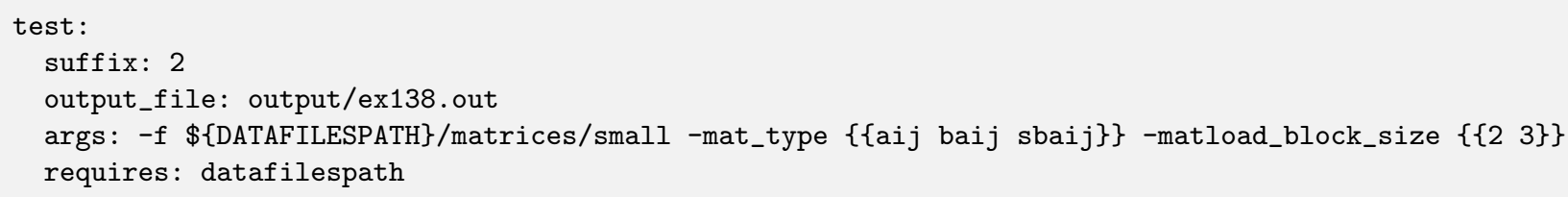

A sample output follows. 
ok 1 In mat...tests: "./ex138 -f \$\{DATAFILESPATH\}/matrices/small -mat_type aij -matload_block_size 2"

ok 2 In mat...tests: "Diff of ./ex138 -f \$\{DATAFILESPATH\}/matrices/small -mat_type aij -matload_block_size 2"

ok 3 In mat...tests: "./ex138 -f \$\{DATAFILESPATH\}/matrices/small -mat_type aij -matload_block_size 3"

ok 4 In mat...tests: "Diff of ./ex138 -f \$\{DATAFILESPATH\}/matrices/small -mat_type aij -matload_block_size 3"

ok 5 In mat...tests: "./ex138 -f \$\{DATAFILESPATH\}/matrices/small -mat_type baij -matload_block_size 2"

ok 6 In mat...tests: "Diff of ./ex138 -f $\$$ \{DATAFILESPATH\}/matrices/small -mat_type baij -matload_block_size 2"

$\ldots$

ok 11 In mat...tests: "./ex138 -f \$\{DATAFILESPATH\}/matrices/small -mat_type saij -matload_block_size 2"

ok 12 In mat...tests: "Diff of ./ex138 -f \$\{DATAFILESPATH\}/matrices/small -mat_type aij -matload_block_size 2"

\subsection{Test Harness Implementation}

Most of the requirements for being TAP-compliant lie in the shell scripts, so we focus on that description.

A sample shell script is given the following.

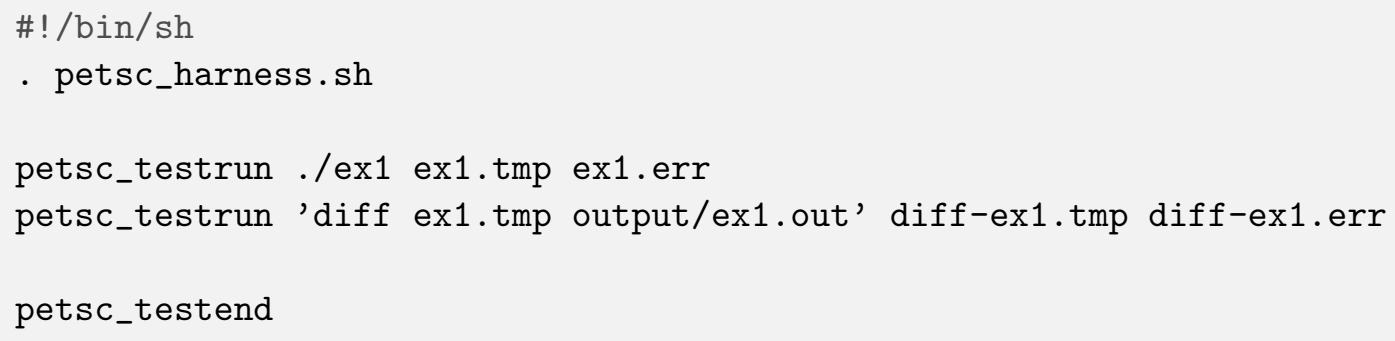

petsc_harness.sh is a small shell script that provides the logging and reporting functions petsc_testrun and petsc_testend.

A small sample of the output from the test harness is as follows.

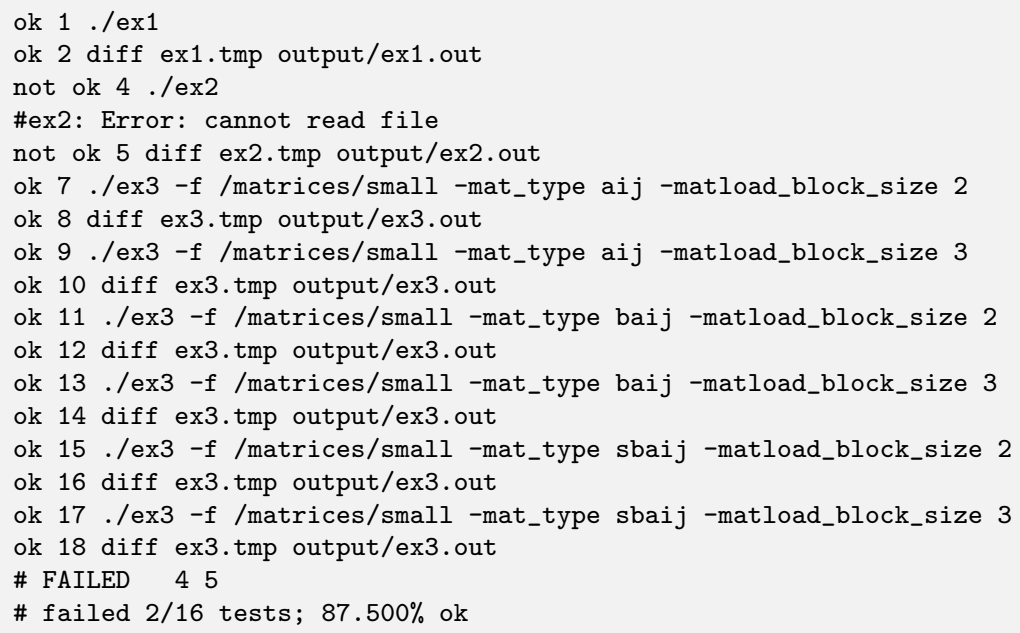

For developers, modifying the lines that get written to the file can be done by modifying \$\{PETSC_DIR\}/config/example_template.py

To modify the test harness, you can modify the following file: 


\subsection{Using the Test Harness for Regression Testing}

The test system can also be helpful for contributing developers in regression testing. A typical workflow might proceed as follows. Note that the exact syntax of all input and output in this section is subject to change.

1. Run the full test suite on the development machine, using the branch of PETSc you want to base your changes on (usually master).

git checkout master

make -f gmakefile test

Confirm that all the tests pass.

2. Create a new branch, and make your changes.

git checkout -b myname/my-feature

\# make and commit changes

3. Run the full test suite on your branch ${ }^{1}$.

make -f gmakefile test

4. Examine the output for any failed tests, for example,

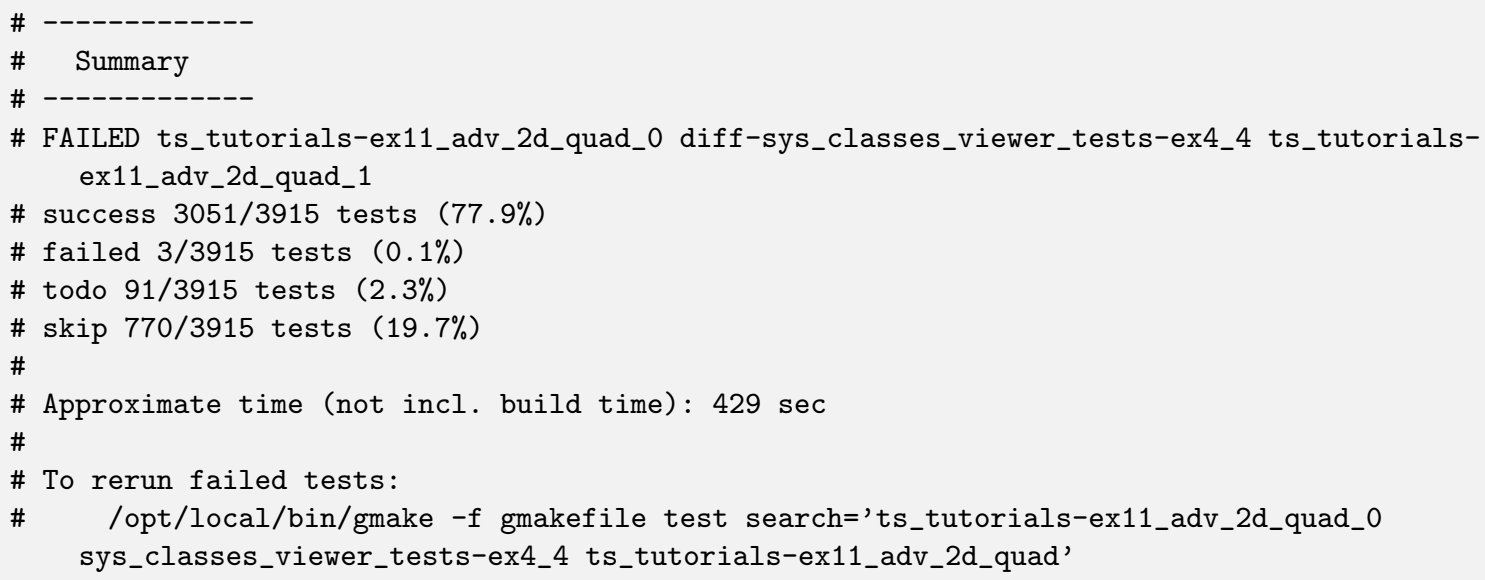

This output indicates that three tests have failed and should be investigated.

5. Rerun one of the failed tests, with additional verbosity.

/opt/local/bin/gmake V=1 -f gmakefile test search=' sys_classes_viewer_tests-ex4_4'

6. Examine the output.

arch-darwin-master-double-debug/tests/sys/classes/viewer/examples/tests/runex4_4.sh -v ok sys_classes_viewer_tests-ex4_4 cat ex4_4.tmp $\mid>/$ dev/null; cat ex4a1.tmp > ex4_4.tmp.tmp 2>> runex4_4.err \&\& mv ex4_4.tmp.tmp ex4_4.tmp

\footnotetext{
${ }^{1}$ For information on how to run a subset of tests, consult the users manual and other sources referenced therein.
} 


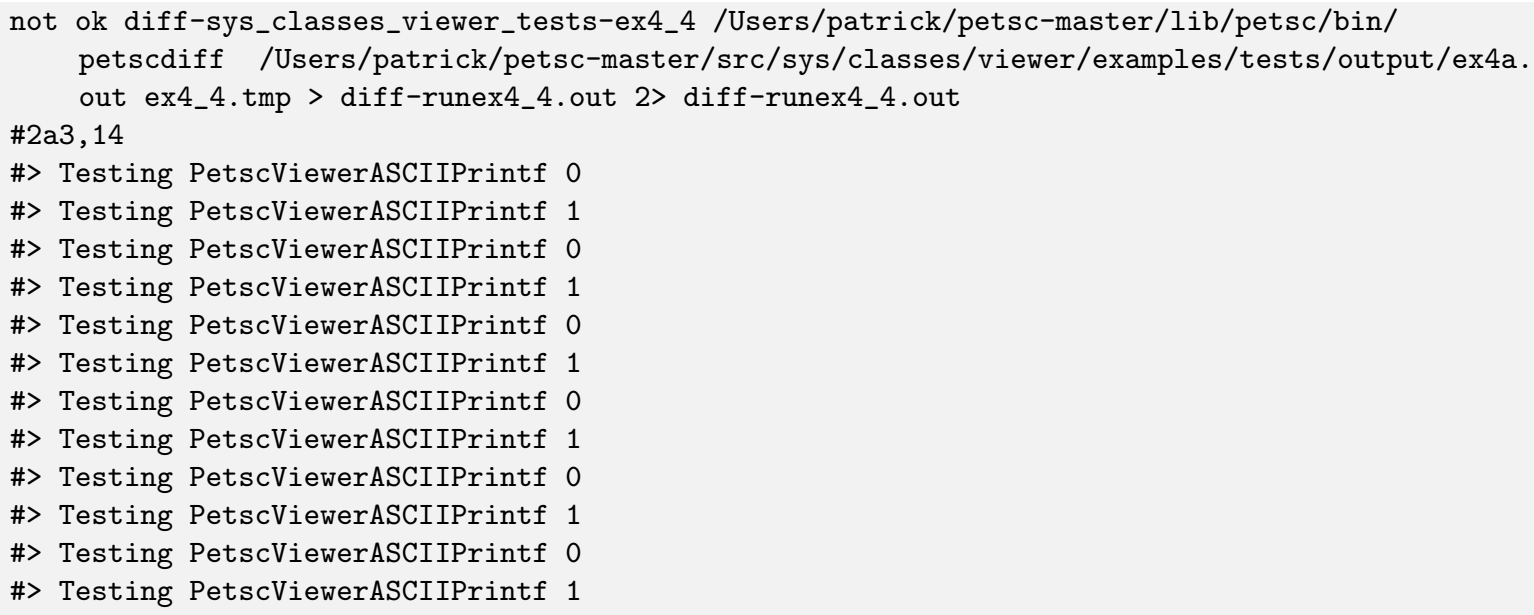

This indicates that the output does not match the reference output.

7. Look in the directory from which the test was run.

cd \$PETSC_ARCH/tests/sys/classes/viewer/examples/tests/

8. The name of the test indicates that the test was defined in ex4.c, with the suffix 4 . This is defined in ex4.c as such:

test:

suffix: 4

args: -myviewer ascii:ex4a1.tmp: :append

filter: cat ex4a1.tmp

output_file: output/ex4a.out

(Note that the default number of MPI processes is 1.)

9. We can deduce now that the command to rerun the example is

make ex4

\$PETSC_DIR/\$PETSC_ARCH/bin/mpiexec -n 1 ./ex4 -myviewer ascii:ex4a1.tmp: : append cat ex4a1.tmp

10. Similarly, we can deduce that the reference output is in \$PETSC_DIR/src/sys/classes/viewer/examples/tests/output/ex4a.out

(Note that there is a default output location in output/testname_suffix.)

11. This should provide enough information to iteratively debug the problem by making changes, recompiling, and rerunning this example directly.

12. Repeat for other errors.

\subsubsection{Additional Tips}

To rerun just the reporting use config/report_tests.py

To see the full options use 
config/report_tests.py -h

To see the full timing information for the five most expensive tests use

config/report_tests.py -t 5 


\section{Bibliography}

[1] S. Balay, W. D. Gropp, L. C. McInnes, and B. F. Smith. CRPC Handbook of Parallel Computing, chapter Software for the Scalable Solution of PDEs. Morgan Kaufmann Publishers, 2002 .

[2] Satish Balay, Shrirang Abhyankar, Mark F. Adams, Jed Brown, Peter Brune, Kris Buschelman, Lisandro Dalcin, Victor Eijkhout, William D. Gropp, Dinesh Kaushik, Matthew G. Knepley, Dave A. May, Lois Curfman McInnes, Richard Tran Mills, Todd Munson, Karl Rupp, Patrick Sanan, Barry F. Smith, Stefano Zampini, Hong Zhang, and Hong Zhang. PETSc users manual. Technical Report ANL-95/11 - Revision 3.9, Argonne National Laboratory, 2018.

[3] Satish Balay, Jed Brown, Matthew G. Knepley, Lois McInnes, and Barry Smith. Software Engineering for Science, chapter Providing Mixed Language and Legacy Support within a Library. Taylor \& Francis, 2015.

[4] Satish Balay, William D. Gropp, Lois Curfman McInnes, and Barry F. Smith. Efficient management of parallelism in object oriented numerical software libraries. In E. Arge, A. M. Bruaset, and H. P. Langtangen, editors, Modern Software Tools in Scientific Computing, pages 163-202. Birkhäuser Press, 1997.

[5] Satish Balay, William D. Gropp, Lois Curfman McInnes, and Barry F. Smith. A microkernel design for component-based parallel numerical software systems. In Proceedings of the SIAM Workshop on Object Oriented Methods for Inter-operable Scientific and Engineering Computing, pages 58-67. SIAM, 1998.

[6] W Gropp. Users manual for bfort: Producing Fortran interfaces to C source code. Technical Report ANL/MCS-TM-208, Argonne National Laboratory, 1995.

[7] W Gropp. Users manual for doctext: Producing documentation from source code. Technical Report ANL/MCS-TM-206, Argonne National Laboratory, 1995.

[8] William D. Gropp and Barry F. Smith. Scalable, extensible, and portable numerical libraries. In Proceedings of the Scalable Parallel Libraries Conference, pages 87-93, Mississippi State University, 1994. IEEE.

[9] Matthew G. Knepley, Jed Brown, Lois Curfman McInnes, and Barry F. Smith. Accurately citing software and algorithms used in publications. In First Workshop on Sustainable Software for Science: Practice and Experiences (WSSSPE), held at SC13, 2013.

[10] B. Smith. Encyclopedia of Parallel Computing, chapter PETSc, the Portable, Extensible Toolkit for Scientific computing. Springer, 2011. 


\section{Argonne}

\section{Mathematics and Computer Science Division}

Argonne National Laboratory

9700 South Cass Avenue, Bldg. 240

Argonne, IL 60439

www.anl.gov 